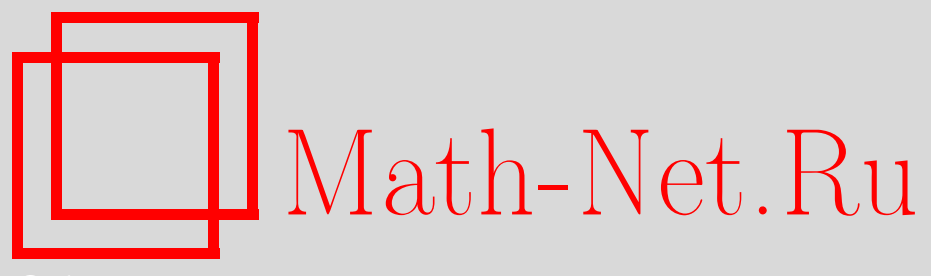

А. Ю. Колесов, Н. Х. Розов, Автоволновые процессы в цепочках диффузионно связанных уравнений с запаздыванием, УМН, 2012, том 67, выпуск 2, 109-156

DOI: https://doi.org/10.4213/rm9469

Использование Общероссийского математического портала Math-Net.Ru подразумевает, что вы прочитали и согласны с пользовательским соглашением http://www . mathnet.ru/rus/agreement

Параметры загрузки:

IP : 52.6 .47 .48

26 апреля 2023 г., 18:01:13

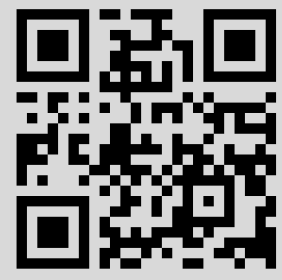




\title{
Автоволновые процессы в цепочках диффузионно связанных уравнений с запаздыванием
}

\author{
А. Ю. Колесов, Н. Х. Розов
}

\begin{abstract}
В настоящей работе вводится в рассмотрение новый математический объект - скалярное нелинейное дифференциально-разностное уравнение с запаздыванием, представляющее собой некоторую модификацию известного уравнения Хатчинсона из экологии. Устанавливается, что в одномерной цепочке диффузионно связанных уравнений такого типа наблюдается известное явление буферности. А именно, при согласованном увеличении числа звеньев рассматриваемой цепочки и уменьшении коэффициента диффузии происходит неограниченный рост количества ее сосуществующих устойчивых периодических движений.

Библиография: 15 названий.
\end{abstract}

Ключевые слова: модифицированное уравнение Хатчинсона, автоволновые процессы, релаксационный цикл, асимптотика, устойчивость.

\section{СОДЕРЖАНИЕ}

1. Введение............................................. 110

2. Модифицированное уравнение Хатчинсона .................. 112

2.1. Основной результат . . . . . . . . . . . . . . . . . . . . 112

2.2. Доказательство существования релаксационного цикла ....... 114

2.3. Анализ свойств устойчивости . . . . . . . . . . . . . . . . . . 120

3. Автоволновые режимы билокальной модели ...................... 122

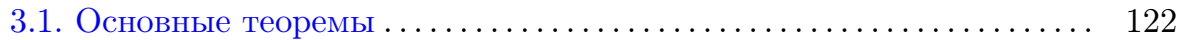

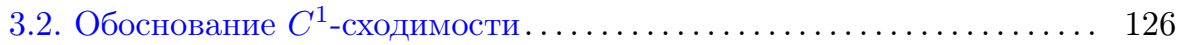

3.3. Анализ предельного отображения ...................... 136

4. Многомерный случай.................................... 143

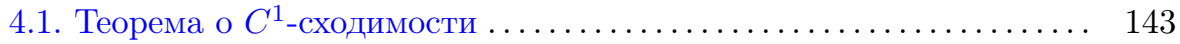

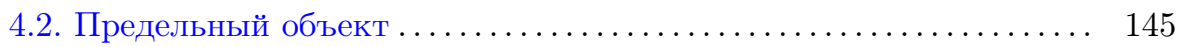

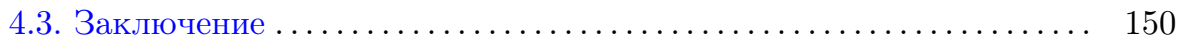

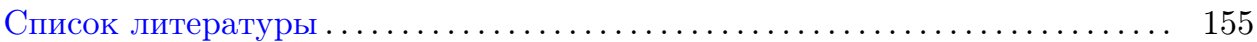

Работа выполнена при финансовой поддержке РФФИ (гранты № 05-01-01004, 09-0100614a) и целевой программы "Научные и научно-педагогические кадры инновационной России" (государственный контракт № 02.740.11.0197). 


\section{1. Введение}

Теория релаксационных колебаний в многомерных системах обыкновенных дифференциальных уравнений берет свое начало с заметки Л. С. Понтрягина и Е. Ф. Мищенко [1], послужившей основой для последующих работ (см. [2]-[5]). $\mathrm{K}$ настоящему времени эта теория приняла достаточно законченный характер и в суммированном виде содержится в монографиях [6], [7]. Необходимо также отметить монографию [8] и статью [9], в которых основные идеи и методы из [6], [7] были перенесены на некоторые классы сингулярно возмущенных дифференциально-разностных уравнений с запаздыванием. Что же касается излагаемых в данной работе результатов, то их можно рассматривать как продолжение начатых в [8], [9] исследований.

Приступим к описанию объектов дальнейшего анализа. В связи с этим обратимся к базовому экологическому уравнению

$$
\dot{N}=\lambda[1-N(t-1)] N
$$

где $N(t) \geqslant 0$ - плотность численности популяции млекопитающих в текущий момент времени, $\lambda=$ const $>0$ - мальтузианский коэффициент линейного роста. Это уравнение было предложено в 1948 г. американским биологом Дж.Э. Хатчинсоном [10] и позднее получило его имя.

Как известно (см. [11]-[13]), при всех $\lambda>\pi / 2$ уравнение (1.1) имеет медленно осциллирующий цикл $N_{*}(t, \lambda)>0, N_{*}(t, \pi / 2) \equiv 1, N_{*}(0, \lambda) \equiv 1$, периода $T_{*}(\lambda)$, $T_{*}(\pi / 2)=4$ (медленная осцилляция означает, что расстояние между любыми соседними нулями функции $N_{*}(t, \lambda)-1$ больше единицы). Упомянутый цикл

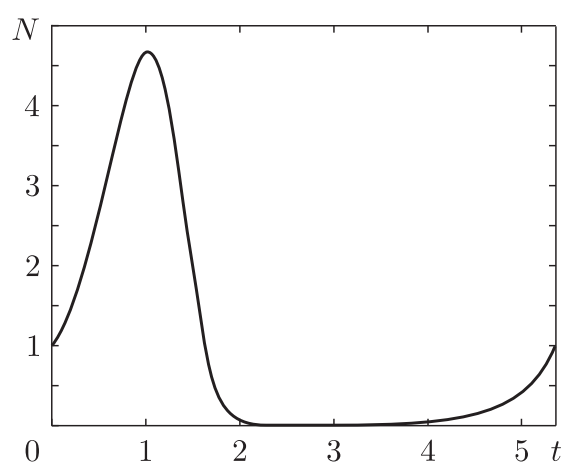

Рис. 1.1

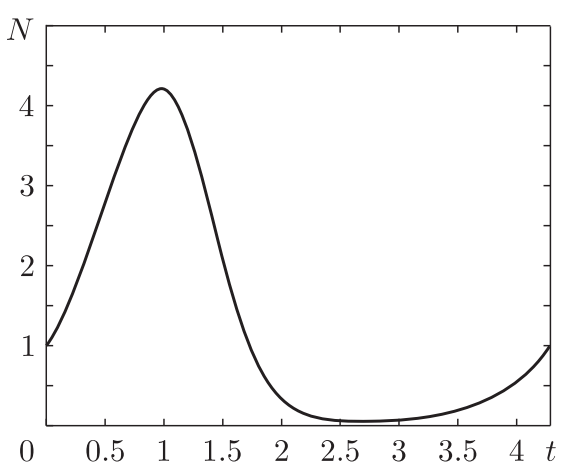

Рис. 1.2

рождается из состояния равновесия $N \equiv 1$ при $0<\lambda-\pi / 2 \ll 1$ в результате бифуркации Андронова-Хопфа, а при последующем увеличении $\lambda$ довольно быстро приобретает релаксационную форму (см. рис. 1.1, где показан график $N_{*}(t, \lambda)$ при $\left.\lambda=2.5\right)$. В случае же $\lambda \gg 1$ справедливы следующие асимптоти- 
ческие равенства [8]:

$$
\begin{gathered}
\max _{0 \leqslant t \leqslant T_{*}} N_{*}(t, \lambda)=\exp (\lambda-1)+(2 e)^{-1}+O(\exp (-\lambda)), \\
\min _{0 \leqslant t \leqslant T_{*}} N_{*}(t, \lambda)=\exp \left[-\exp \lambda+2 \lambda-1+\frac{1+(1+\lambda) \ln \lambda}{\lambda}+O\left(\frac{\ln ^{2} \lambda}{\lambda^{2}}\right)\right], \\
T_{*}(\lambda)=\frac{1+\exp \lambda}{\lambda}+O\left(\frac{\exp (-\lambda)}{\lambda}\right) .
\end{gathered}
$$

Добавим еще, что, как показывают численные эксперименты, цикл $N_{*}(t, \lambda)$ является экспоненциально орбитально устойчивым (в метрике фазового пространства $C[-1,0])$ при всех $\lambda>\pi / 2$. Однако строго доказать этот факт удается лишь при $0<\lambda-\pi / 2 \ll 1$ и $\lambda \gg 1$.

Из формул (1.2)-(1.4) вытекает, что при больших $\lambda$ закон $N_{*}(t, \lambda)$ изменения плотности популяции биологически нереализуем. Действительно, излишне большой период колебаний и излишне глубокий минимум фактически означают, что после первого же всплеска численности произойдет полное вымирание вида и следующий всплеск просто не наступит. В связи с этим возникает необходимость в подходящей модификации уравнения (1.1), улучшающей биологические характеристики его релаксационного цикла. Одна из таких модификаций предлагается в настоящей статье и заключается в следующем.

Рассмотрим вместо (1.1) уравнение вида

$$
\dot{N}=\lambda f(N(t-1)) N
$$

Здесь, как и выше, параметр $\lambda$ предполагается большим, а бесконечно дифференцируемая на полуоси $\mathbb{R}_{+}=\{x \in \mathbb{R}: x \geqslant 0\}$ функция $f(x)$ обладает свойствами

$$
f(0)=1, \quad f(x)=-a_{0}+\sum_{k=1}^{\infty} \frac{a_{k}}{x^{k}}, \quad x \rightarrow+\infty, \quad a_{0}>0 .
$$

Считаем еще, что фигурирующий в (1.6) асимптотический ряд можно дифференцировать по $x$ любое число раз. Типовым примером такой функции служит

$$
f(x)=\frac{1-x}{1+c x}, \quad c=\text { const }>0 .
$$

Как будет показано ниже, замена уравнения (1.1) на (1.5) приводит к желаемой цели: модифицированное уравнение Хатчинсона (1.5) допускает устойчивый релаксационный цикл $N_{*}(t, \lambda)>0, \quad N_{*}(0, \lambda) \equiv 1$ периода $T_{*}(\lambda)$ : $\lim T_{*}(\lambda)=T_{0}, \lambda \rightarrow \infty$, где

$$
T_{0}=2+a_{0}+\frac{1}{a_{0}} .
$$

Что же касается формул (1.2), (1.3), то теперь их аналоги имеют вид

$$
\max _{t} N_{*}(t, \lambda) \sim c_{1} \exp \lambda, \quad \min _{t} N_{*}(t, \lambda) \sim c_{2} \exp \left(-a_{0} \lambda\right)
$$


где $c_{1}, c_{2}=$ const $>0$. Таким образом, налицо требуемое улучшение биологических характеристик релаксационных автоколебаний уравнения (1.5) по сравнению с уравнением (1.1). Более того, сопоставляя рис. 1.1 с графиком цикла $N_{*}(t, \lambda)$ уравнения (1.5), (1.7) при $\lambda=2.5, c=0.2$ (см. рис. 1.2), убеждаемся, что это улучшение проявляется даже при $\lambda \sim 1$.

Уравнение (1.5) описывает динамику изменения плотности популяции, обитающей в однородном ареале, когда пищевая база регулярно восстанавливается до некоторого фиксированного уровня, а миграционный фактор столь велик, что пространственные возмущения гаснут. При этих биологических посылках рассмотрим цепочку из $m$ локальных ареалов, соединенных узкими проходами. В результате придем к системе

$$
\dot{N}_{j}=d\left(N_{j+1}-2 N_{j}+N_{j-1}\right)+\lambda f\left(N_{j}(t-1)\right) N_{j}, \quad j=1, \ldots, m,
$$

где $N_{0}=N_{1}, N_{m+1}=N_{m}, \lambda \gg 1$, а параметр $d$, имеющий порядок единицы, характеризует глубину связи между ареалами.

Система (1.10) допускает, очевидно, так называемый однородный, или синхронный, цикл

$$
N_{1} \equiv \cdots \equiv N_{m}=N_{*}(t, \lambda),
$$

где $N_{*}(t, \lambda)$ - устойчивое периодическое решение уравнения (1.5). Наш основной результат состоит в том, что при подходящем уменьшении $d$ и при всех $\lambda \gg 1$ эта система наряду с устойчивым циклом (1.11) имеет не менее $m$ экспоненциально орбитально устойчивых неоднородных периодических движений, которые по аналогии с пространственно непрерывным случаем будем называть автоволновыми процессами.

\section{2. Модифицированное уравнение Хатчинсона}

2.1. Основной результат. При исследовании вопросов о существовании у уравнения (1.5) релаксационного цикла $N_{*}(t, \lambda)$ с требуемыми характеристиками (1.9) и об устойчивости этого цикла удобно сделать в (1.5) замену $N=\exp (\lambda x)$. Действительно, указанная замена преобразует интересующее нас уравнение к виду

$$
\dot{x}=F(x(t-1), \varepsilon),
$$

где $F(x, \varepsilon)=f(\exp (x / \varepsilon)), \varepsilon=1 / \lambda \ll 1$. Далее, свойства (1.6) функции $f(x)$ позволяют заключить, что

$$
\lim _{\varepsilon \rightarrow 0} F(x, \varepsilon)=R(x), \quad R(x)= \begin{cases}1 & \text { при } x<0, \\ -a_{0} & \text { при } x>0 .\end{cases}
$$

Таким образом, исходному сингулярно возмущенному уравнению (2.1) ставится в соответствие предельное релейное уравнение с запаздыванием

$$
\dot{x}=R(x(t-1)) .
$$

Как и в работе [9], понятие решения уравнения (2.2) определим конструктивно. С этой целью зафиксируем некоторое

$$
\sigma_{0}: 0<\sigma_{0}<a_{0},
$$




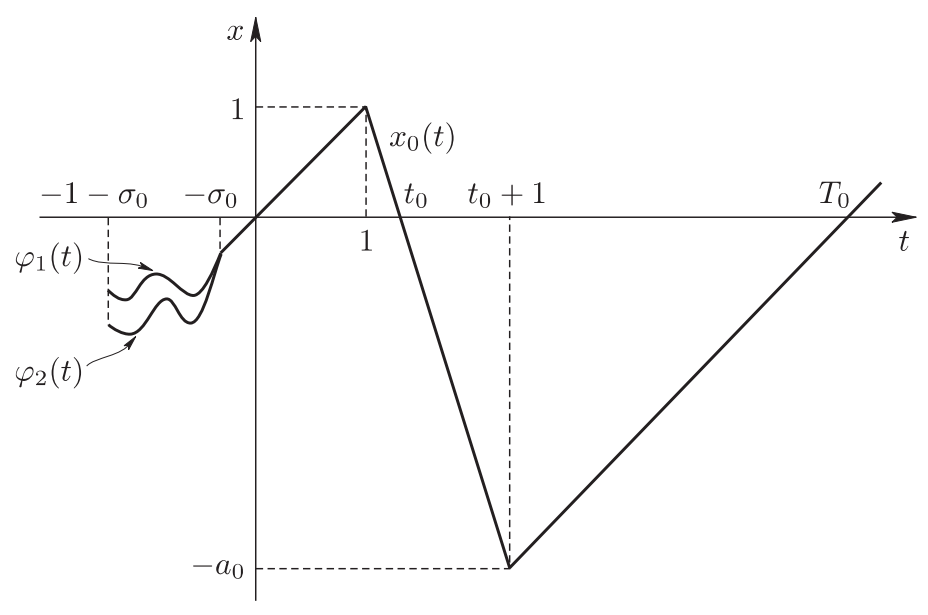

Рис. 2.1

рассмотрим множество функций

$$
\varphi(t) \in C\left[-1-\sigma_{0},-\sigma_{0}\right], \quad \varphi(t)<0 \quad \forall t \in\left[-1-\sigma_{0},-\sigma_{0}\right], \quad \varphi\left(-\sigma_{0}\right)=-\sigma_{0}
$$

и обозначим через $x_{\varphi}(t), t \geqslant-\sigma_{0}$, решение уравнения $(2.2)$ с произвольной начальной функцией (2.4). Заметим, далее, что поскольку $\varphi(t-1)<0$ при $t \in\left[-\sigma_{0}, 1-\sigma_{0}\right]$, то на указанном промежутке времени, согласно $(2.2), x_{\varphi}(t)$ является решением задачи Коши $\dot{x}=1, x\left(-\sigma_{0}\right)=-\sigma_{0}$ и, следовательно,

$$
x_{\varphi}(t)=t
$$

Ясно также, что формула (2.5) сохраняется до тех пор, пока $x_{\varphi}(t-1)<0$. Тем самым, она справедлива на полуинтервале $-\sigma_{0} \leqslant t<1$.

При $t=1$ первый раз происходит переключение, и при $t>1$ решение $x_{\varphi}(t)$ определяется уже из задачи Коши $\dot{x}=-a_{0}, x(1)=1$, т. е. посредством равенства

$$
x_{\varphi}(t)=1-a_{0}(t-1) .
$$

В свою очередь, формула (2.6) остается в силе, пока $x_{\varphi}(t-1)>0$, т. е. до очередного момента переключения $t=t_{0}+1$, где

$$
t_{0}=1+\frac{1}{a_{0}} .
$$

На промежутке же $t_{0}+1 \leqslant t \leqslant T_{0}-\sigma_{0}$, где $T_{0}$ определено формулой (1.8), функция $x_{\varphi}(t)$ задается аналогичным (2.5) равенством

$$
x_{\varphi}(t)=-a_{0}+t-t_{0}-1 \text {. }
$$

Завершая описание построения решения $x_{\varphi}(t)$, отметим, что в силу условия (2.3) на постоянную $\sigma_{0}$ функция $x_{\varphi}\left(t+T_{0}\right),-1-\sigma_{0} \leqslant t \leqslant-\sigma_{0}$, принадлежит исходному начальному множеству (2.4). А это означает, что при $t \geqslant T_{0}-\sigma_{0}$ 
весь процесс повторяется. Более того, из (2.6)-(2.8) вытекает, что каждое решение $x_{\varphi}(t)$ с начальным условием (2.4) при всех $t \geqslant-\sigma_{0}$ совпадает с одной и той же $T_{0}$-периодической функцией (см. рис. 2.1 )

$$
x_{0}(t)=\left\{\begin{array}{ll}
t & \text { при } 0 \leqslant t \leqslant 1, \\
1-a_{0}(t-1) & \text { при } 1 \leqslant t \leqslant t_{0}+1, \\
-a_{0}+t-t_{0}-1 & \text { при } t_{0}+1 \leqslant t \leqslant T_{0},
\end{array} \quad x_{0}\left(t+T_{0}\right) \equiv x_{0}(t) .\right.
$$

Перейдем к вопросу о связи между периодическими решениями уравнений (2.1) и (2.2). Справедливо следующее утверждение.

Теорема 2.1. При всех достаточно малых $\varepsilon>0$ уравнение (2.1) имеет единственный орбитально экспоненциально устойчивый цикл $x_{*}(t, \varepsilon)$ периода $T_{*}(\varepsilon)$, удовлетворяющий тождеству $x_{*}\left(-\sigma_{0}, \varepsilon\right) \equiv-\sigma_{0}$ и предельным равенствам

$$
\lim _{\varepsilon \rightarrow 0} \max _{0 \leqslant t \leqslant T_{*}(\varepsilon)}\left|x_{*}(t, \varepsilon)-x_{0}(t)\right|=0, \quad \lim _{\varepsilon \rightarrow 0} T_{*}(\varepsilon)=T_{0} .
$$

Доказательство сформулированной теоремы, содержащееся в следующих двух пунктах, опирается на некоторые дополнительные конструкции. Для их описания зафиксируем наряду с константой $\sigma_{0}$ (см. (2.3)) постоянные $q_{1}>\sigma_{0}$, $q_{2} \in\left(0, \sigma_{0}\right)$ и обозначим через $S\left(\sigma_{0}, q_{1}, q_{2}\right) \subset C\left[-1-\sigma_{0},-\sigma_{0}\right]$ замкнутое, ограниченное и выпуклое множество функций $\varphi(t)$, удовлетворяющих требованиям

$$
-q_{1} \leqslant \varphi(t) \leqslant-q_{2}, \quad \varphi\left(-\sigma_{0}\right)=-\sigma_{0} .
$$

Далее, для произвольной функции $\varphi \in S\left(\sigma_{0}, q_{1}, q_{2}\right)$ рассмотрим решение $x=$ $x_{\varphi}(t, \varepsilon), t \geqslant-\sigma_{0}$, уравнения $(2.1)$ с начальным условием $\varphi(t),-1-\sigma_{0} \leqslant$ $t \leqslant-\sigma_{0}$, а через $t=T_{\varphi}$ обозначим второй положительный корень уравнения $x_{\varphi}\left(t-\sigma_{0}, \varepsilon\right)=-\sigma_{0}$ (если он существует). И наконец, зададим оператор $\Pi_{\varepsilon}: S\left(\sigma_{0}, q_{1}, q_{2}\right) \rightarrow C\left[-1-\sigma_{0},-\sigma_{0}\right]$ с помощью равенства

$$
\Pi_{\varepsilon}(\varphi)=x_{\varphi}\left(t+T_{\varphi}, \varepsilon\right), \quad-1-\sigma_{0} \leqslant t \leqslant-\sigma_{0} .
$$

План последующих действий таков. Сначала мы установим асимптотические формулы для $x_{\varphi}(t, \varepsilon)$ на различных промежутках изменения $t$, из которых будет следовать, что при подходящем выборе параметров $\sigma_{0}, q_{1}, q_{2}$ оператор (2.12) определен на множестве $S\left(\sigma_{0}, q_{1}, q_{2}\right)$ и преобразует его в себя. Затем проведем анализ уравнения в вариациях на решении $x_{\varphi}(t, \varepsilon)$ и покажем, что $\Pi_{\varepsilon}$ является сжимающим.

2.2. Доказательство существования релаксационного цикла. Построение асимптотики функции $x_{\varphi}(t, \varepsilon)$ начнем с отрезка $-\sigma_{0} \leqslant t \leqslant 1-\sigma_{0}$, на котором согласно (2.1) она является решением задачи Коши

$$
\dot{x}=F(\varphi(t-1), \varepsilon),\left.\quad x\right|_{t=-\sigma_{0}}=-\sigma_{0} .
$$

Далее, опираясь на неравенства из (2.11) и свойства (1.6), замечаем, что равномерно по $t \in\left[-\sigma_{0}, 1-\sigma_{0}\right], \varphi \in S\left(\sigma_{0}, q_{1}, q_{2}\right)$

$$
F(\varphi(t-1), \varepsilon)=1+O\left(\exp \left(-\frac{q_{2}}{\varepsilon}\right)\right) .
$$


А отсюда очевидным образом выводим, что

$$
x_{\varphi}(t, \varepsilon)=t+\Delta_{1, \varphi}(t, \varepsilon), \quad-\sigma_{0} \leqslant t \leqslant 1-\sigma_{0} ;
$$

здесь и далее через $\Delta_{1, \varphi}, \Delta_{2, \varphi}$ и т. д. обозначаются остатки, модули которых имеют экспоненциальный порядок малости (т. е. порядок $\exp (-q / \varepsilon), q=$ const $>0)$ равномерно по $\varphi, t$.

На следующем этапе, считая, что

$$
\sigma_{0}<\frac{1}{2}
$$

обратимся к отрезку $1-\sigma_{0} \leqslant t \leqslant 1+\sigma_{0}$. Так как в силу (2.15) его длина не превосходит единицы, то в этом случае $x_{\varphi}(t-1, \varepsilon)$ задается уже известным равенством (2.14) (при замене в нем $t$ на $t-1$ ). Тем самым, и здесь имеем дело с задачей Коши

$$
\dot{x}=F\left(t-1+\Delta_{1, \varphi}(t-1, \varepsilon), \varepsilon\right),\left.\quad x\right|_{t=1-\sigma_{0}}=1-\sigma_{0}+\Delta_{1, \varphi}\left(1-\sigma_{0}, \varepsilon\right),
$$

правые части которой известны из предыдущего шага. Что же касается решения этой задачи, то его будем искать в виде

$$
x_{\varphi}(t, \varepsilon)=1+\left.\varepsilon v(\tau)\right|_{\tau=(t-1) / \varepsilon}+\Delta_{2, \varphi}(t, \varepsilon), \quad 1-\sigma_{0} \leqslant t \leqslant 1+\sigma_{0},
$$

где

$$
v(\tau)=\tau+\int_{-\infty}^{\tau}[f(\exp s)-1] d s,
$$

a $\Delta_{2, \varphi}$ - подлежащий определению остаток.

Подставляя выражения $(2.17),(2.18)$ в $(2.16)$, для $\Delta_{2, \varphi}$ приходим к задаче Коши

$$
\dot{\Delta}_{2, \varphi}=G_{\varphi}(t, \varepsilon),\left.\quad \Delta_{2, \varphi}\right|_{t=1-\sigma_{0}}=g_{\varphi}(\varepsilon),
$$

где

$$
\begin{aligned}
G_{\varphi}(t, \varepsilon)= & f\left(x_{1}\right)-f\left(x_{2}\right) \\
& \text { при } \quad x_{1}=\exp \left(\frac{t-1}{\varepsilon}+\frac{\Delta_{1, \varphi}(t-1, \varepsilon)}{\varepsilon}\right), \quad x_{2}=\exp \left(\frac{t-1}{\varepsilon}\right) ; \\
g_{\varphi}(\varepsilon)= & -\sigma_{0}-\left.\varepsilon v(\tau)\right|_{\tau=-\sigma_{0} / \varepsilon}+\Delta_{1, \varphi}\left(1-\sigma_{0}, \varepsilon\right) .
\end{aligned}
$$

Таким образом, доказательство равномерной по $\varphi, t$ экспоненциальной малости $\Delta_{2, \varphi}$ сводится к проверке аналогичных свойств для функции (2.20) и начального условия (2.21).

Обратимся сначала к анализу $g_{\varphi}(\varepsilon)$. Учитывая в $(2.18)$ первое из соотношений (1.6), убеждаемся, что

$$
v(\tau)=\tau+O(\exp \tau), \quad \tau \rightarrow-\infty
$$

и, следовательно,

$$
-\sigma_{0}-\left.\varepsilon v(\tau)\right|_{\tau=-\sigma_{0} / \varepsilon}=O\left(\exp \left(-\frac{\sigma_{0}}{\varepsilon}\right)\right) .
$$


А отсюда и из известных свойств $\Delta_{1, \varphi}$ вытекает требуемая экспоненциальная малость величины (2.21).

Для оценки функции (2.20) продифференцируем по $x$ асимптотическое разложение из (1.6). В результате приходим к выводу, что

$$
M_{1}=\sup _{x \in \mathbb{R}_{+}}\left(1+x^{2}\right)\left|f^{\prime}(x)\right|<\infty .
$$

Далее, используя этот факт, нетрудно увидеть, что

$$
\left|f\left(x_{1}\right)-f\left(x_{2}\right)\right| \leqslant \frac{M_{1}}{1+\min \left(x_{1}^{2}, x_{2}^{2}\right)}\left|x_{1}-x_{2}\right| \quad \forall x_{1}, x_{2} \in \mathbb{R}_{+} .
$$

Применяя затем свойство (2.23) к правой части из (2.20), получаем неравенство

$$
\left|G_{\varphi}(t, \varepsilon)\right| \leqslant \frac{M_{2}}{\varepsilon} \exp \left(-\frac{|t-1|}{\varepsilon}\right)\left|\Delta_{1, \varphi}(t-1, \varepsilon)\right|, \quad 1-\sigma_{0} \leqslant t \leqslant 1+\sigma_{0},
$$

где $M_{2}$ - некоторая универсальная (не зависящая от $t, \varphi, \varepsilon$ ) положительная константа. Остается заметить, что из (2.24) равномерная по $t, \varphi$ экспоненциальная малость $G_{\varphi}(t, \varepsilon)$ следует уже очевидным образом.

Дальнейший анализ связан с рассмотрением отрезка $1+\sigma_{0} \leqslant t \leqslant 2+\sigma_{0}$ при условии

$$
\sigma_{0}<\frac{1}{2 a_{0}}
$$

гарантирующем выполнение неравенства $2+\sigma_{0}<t_{0}+1-\sigma_{0}$, где, напомним, $t_{0}$ - величина (2.7). В этом случае на промежутках $t \in\left[1+\sigma_{0}, 2-\sigma_{0}\right]$ и $t \in$ $\left[2-\sigma_{0}, 2+\sigma_{0}\right]$ функция $x_{\varphi}(t-1, \varepsilon)$ задается соответственно формулами $(2.14)$ и $(2.17)$ (с заменой в них $t$ на $t-1)$. А отсюда, в частности, следует, что

$$
x_{\varphi}(t-1, \varepsilon) \geqslant q, \quad t \in\left[1+\sigma_{0}, 2-\sigma_{0}\right],
$$

где $q$ - произвольно фиксированная постоянная из интервала $\left(0, \sigma_{0}\right)$.

Для получения аналогичной (2.26) оценки на отрезке $2-\sigma_{0} \leqslant t \leqslant 2+\sigma_{0}$ необходимо наряду с (2.22) знать поведение функции (2.18) при $\tau \rightarrow+\infty$. Несложный подсчет показывает, что

$$
v(\tau)=-a_{0} \tau+c_{0}+O(\exp (-\tau)), \quad \tau \rightarrow+\infty,
$$

где

$$
c_{0}=\int_{0}^{1} \frac{f(u)-1}{u} d u+\int_{1}^{+\infty} \frac{f(u)+a_{0}}{u} d u .
$$

Учитывая, далее, соотношения (2.22), (2.27), (2.28), из (2.17) делаем вывод, что

$$
x_{\varphi}(t-1, \varepsilon) \geqslant q, \quad t \in\left[2-\sigma_{0}, 2+\sigma_{0}\right],
$$

где теперь $q \in\left(0, \min \left(1-\sigma_{0}, 1-a_{0} \sigma_{0}\right)\right)$.

Установленные выше неравенства (2.26), (2.29) и свойства (1.6) функции $f(x)$ позволяют заключить, что равномерно по $t \in\left[1+\sigma_{0}, 2+\sigma_{0}\right], \varphi \in S\left(\sigma_{0}, q_{1}, q_{2}\right)$ справедливо аналогичное (2.13) асимптотическое представление

$$
F\left(x_{\varphi}(t-1, \varepsilon), \varepsilon\right)=-a_{0}+O\left(\exp \left(-\frac{q}{\varepsilon}\right)\right), \quad q=\text { const }>0 .
$$


Подставляя его вместе с формулами $(2.17),(2.18),(2.27),(2.28)$ в интегральное соотношение

$$
x_{\varphi}(t, \varepsilon)=\left.x_{\varphi}\right|_{t=1+\sigma_{0}}+\int_{1+\sigma_{0}}^{t} F\left(x_{\varphi}(s-1, \varepsilon), \varepsilon\right) d s,
$$

убеждаемся, что

$$
x_{\varphi}(t, \varepsilon)=1-a_{0}(t-1)+\varepsilon c_{0}+\Delta_{3, \varphi}(t, \varepsilon),
$$

где, как обычно, остаток $\Delta_{3, \varphi}$ равномерно по $\varphi, t$ экспоненциально мал.

Добавим еще, что на самом деле формула (2.32) сохраняется (вместе с требуемой малостью остатка) и на отрезке $2+\sigma_{0} \leqslant t \leqslant t_{0}+1-\sigma_{0}$. Обосновать этот факт позволяет так называемый метод шагов, суть которого состоит в следующем. Разобьем указанный отрезок на части длины не более единицы и на очередном промежутке, опираясь на формулу (2.32) (уже установленную на предыдущем участке изменения $t$ ), сначала получаем оценку вида $x_{\varphi}(t-1, \varepsilon) \geqslant q$ с некоторой универсальной постоянной $q>0$, а затем распространяем равенство (2.32) на шаг вперед. Ясно, что описанный процесс продолжается до тех пор, пока $1-a_{0}(t-2)>0$. Тем самым, рассуждая подобным образом, мы заведомо добираемся до точки $t=t_{0}+1-\sigma_{0}$.

На очередном отрезке $t_{0}+1-\sigma_{0} \leqslant t \leqslant t_{0}+1+\sigma_{0}$ в силу $(2.1),(2.32)$ и оценок $(2.15),(2.25)$ интересующее нас решение $x_{\varphi}(t, \varepsilon)$ определяется из задачи Коши

$$
\begin{gathered}
\dot{x}=F\left(-a_{0}\left(t-t_{0}-1\right)+\varepsilon c_{0}+\Delta_{3, \varphi}(t-1, \varepsilon), \varepsilon\right), \\
\left.x\right|_{t=t_{0}+1-\sigma_{0}}=-a_{0}\left(1-\sigma_{0}\right)+\varepsilon c_{0}+\Delta_{3, \varphi}\left(t_{0}+1-\sigma_{0}, \varepsilon\right) .
\end{gathered}
$$

В свою очередь, решение получившейся задачи будем искать в виде

$$
x_{\varphi}(t, \varepsilon)=-a_{0}+\left.\varepsilon w(\tau)\right|_{\tau=\left(t-t_{0}-1\right) / \varepsilon}+\Delta_{4, \varphi}(t, \varepsilon), \quad t_{0}+1-\sigma_{0} \leqslant t \leqslant t_{0}+1+\sigma_{0},
$$

где

$$
w(\tau)=-a_{0} \tau+c_{0}+\int_{-\infty}^{\tau}\left[f\left(\exp \left(-a_{0} s+c_{0}\right)\right)+a_{0}\right] d s .
$$

В результате для очередного остатка $\Delta_{4, \varphi}$ получаем аналогичную (2.19) задачу Коши

$$
\dot{\Delta}_{4, \varphi}=G_{\varphi}(t, \varepsilon),\left.\quad \Delta_{4, \varphi}\right|_{t=t_{0}+1-\sigma_{0}}=g_{\varphi}(\varepsilon),
$$

где теперь

$$
\begin{gathered}
G_{\varphi}(t, \varepsilon)=f\left(x_{1}\right)-f\left(x_{2}\right) \\
\text { при } \quad x_{1}=\exp \left(-\frac{a_{0}\left(t-t_{0}-1\right)}{\varepsilon}+c_{0}+\frac{\Delta_{3, \varphi}(t-1, \varepsilon)}{\varepsilon}\right), \\
x_{2}=\exp \left(-\frac{a_{0}\left(t-t_{0}-1\right)}{\varepsilon}+c_{0}\right) ; \\
g_{\varphi}(\varepsilon)=a_{0} \sigma_{0}+\varepsilon c_{0}-\left.\varepsilon w(\tau)\right|_{\tau=-\sigma_{0} / \varepsilon}+\Delta_{3, \varphi}\left(t_{0}+1-\sigma_{0}, \varepsilon\right) .
\end{gathered}
$$


Оценка правых частей из (2.35) проводится по той же схеме, что и в случае (2.20), (2.21). Действительно, привлекая вытекающее из (2.34) асимптотическое равенство

$$
w(\tau)=-a_{0} \tau+c_{0}+O\left(\exp \left(a_{0} \tau\right)\right), \quad \tau \rightarrow-\infty,
$$

приходим к выводу, что

$$
a_{0} \sigma_{0}+\varepsilon c_{0}-\left.\varepsilon w(\tau)\right|_{\tau=-\sigma_{0} / \varepsilon}=O\left(\exp \left(-\frac{a_{0} \sigma_{0}}{\varepsilon}\right)\right) .
$$

А отсюда с учетом уже установленных свойств $\Delta_{3, \varphi}$ следует нужная экспоненциальная малость начального условия (2.37). Что же касается функции (2.36), то проверка ее равномерной по $t, \varphi$ экспоненциальной малости базируется на неравенстве (2.23) (см. аналогичное место выше).

Перейдем, далее, к отрезку $t_{0}+1+\sigma_{0} \leqslant t \leqslant \min \left(t_{0}+2+\sigma_{0}, T_{0}+\sigma_{0}\right)$. Поскольку его длина не превосходит единицы, то при указанных $t$ функция $x_{\varphi}(t-1, \varepsilon)$ уже известна и задается равенствами (2.32) или (2.33) (при замене в них $t$ на $t-1$ ). В обоих случаях, однако, справедлива оценка вида

$$
x_{\varphi}(t-1, \varepsilon) \leqslant-q
$$

с некоторой универсальной положительной константой $q$.

Действительно, при тех значениях $t$, когда работает формула (2.32), в качестве $q$ в (2.39) можно взять любое число из интервала $\left(0, a_{0} \sigma_{0}\right)$. В случае же, когда имеем дело с формулой (2.33), для оценки $q$ кроме свойства $(2.38)$ необходимо знать асимптотическое поведение функции (2.34) при $\tau \rightarrow+\infty$. Соответствующую асимптотическую формулу легко вывести, исходя из представления

$$
\begin{aligned}
w(\tau)=\tau & -\frac{c_{0}}{a_{0}}+\int_{-\infty}^{c_{0} / a_{0}}\left[f\left(\exp \left(-a_{0} s+c_{0}\right)\right)+a_{0}\right] d s \\
& +\int_{c_{0} / a_{0}}^{+\infty}\left[f\left(\exp \left(-a_{0} s+c_{0}\right)\right)-1\right] d s-\int_{\tau}^{+\infty}\left[f\left(\exp \left(-a_{0} s+c_{0}\right)\right)-1\right] d s .
\end{aligned}
$$

Для этого достаточно заметить, что последнее слагаемое в $(2.40)$ при $\tau \rightarrow+\infty$ имеет порядок $\exp \left(-a_{0} \tau\right)$, а сумма

$$
\begin{gathered}
\int_{-\infty}^{c_{0} / a_{0}}\left[f\left(\exp \left(-a_{0} s+c_{0}\right)\right)+a_{0}\right] d s+\int_{c_{0} / a_{0}}^{+\infty}\left[f\left(\exp \left(-a_{0} s+c_{0}\right)\right)-1\right] d s \\
=\frac{1}{a_{0}}\left(\int_{0}^{1} \frac{f(u)-1}{u} d u+\int_{1}^{+\infty} \frac{f(u)+a_{0}}{u} d u\right)
\end{gathered}
$$

в силу (2.28) совпадает с $c_{0} / a_{0}$. Таким образом, из $(2.40)$ окончательно получаем

$$
w(\tau)=\tau+O\left(\exp \left(-a_{0} \tau\right)\right), \quad \tau \rightarrow+\infty
$$


Возвратимся к оценке (2.39). Из (2.33), (2.38), (2.41) следует, что в случае, когда $x_{\varphi}(t-1, \varepsilon)$ задается с помощью формулы $(2.33)$, в качестве $q$ в $(2.39)$ можно взять любое число из интервала $\left(0, \min \left(a_{0}-\sigma_{0}, a_{0}-a_{0} \sigma_{0}\right)\right)$.

Установленное выше неравенство (2.39) и свойства (1.6) приводят к асимптотическому представлению

$$
F\left(x_{\varphi}(t-1, \varepsilon), \varepsilon\right)=1+O\left(\exp \left(-\frac{q}{\varepsilon}\right)\right), \quad q=\text { const }>0,
$$

которое, как и аналогичные ему соотношения (2.13), (2.30), справедливо равномерно по $\varphi, t$. Дальнейший же способ действий стандартен: подставляем (2.42) вместе с формулами $(2.33),(2.34),(2.41)$ в аналогичное $(2.31)$ интегральное уравнение

$$
x_{\varphi}(t, \varepsilon)=\left.x_{\varphi}\right|_{t=t_{0}+1+\sigma_{0}}+\int_{t_{0}+1+\sigma_{0}}^{t} F\left(x_{\varphi}(s-1, \varepsilon), \varepsilon\right) d s
$$

и после несложных выкладок приходим к очередному асимптотическому равенству

$$
x_{\varphi}(t, \varepsilon)=t-T_{0}+\Delta_{5, \varphi}(t, \varepsilon),
$$

где остаток $\Delta_{5, \varphi}$ обладает требуемой экспоненциальной малостью.

В случае, когда $t_{0}+2<T_{0}$, отдельному рассмотрению подлежит еще отрезок $t_{0}+2+\sigma_{0} \leqslant t \leqslant T_{0}+\sigma_{0}$. Однако здесь не возникают новые моменты, так как при указанных $t$ сохраняется априорная оценка (2.39) (см. аналогичное место при обосновании формулы (2.32)). Тем самым, асимптотическое представление (2.44) оказывается пригодным на всем отрезке $t_{0}+1+\sigma_{0} \leqslant t \leqslant T_{0}+\sigma_{0}$.

Подведем некоторый итог. Из (2.42)-(2.44) очевидным образом следует, что интересующий нас корень $t=T_{\varphi}$ уравнения $x_{\varphi}\left(t-\sigma_{0}, \varepsilon\right)=-\sigma_{0}$ определяется однозначно, причем равномерно по $\varphi \in S\left(\sigma_{0}, q_{1}, q_{2}\right)$

$$
T_{\varphi}=T_{0}+O\left(\exp \left(-\frac{q}{\varepsilon}\right)\right), \quad q=\text { const }>0 .
$$

Далее, объединяя все полученные выше асимптотические представления для решения $x_{\varphi}(t, \varepsilon)$ (см. $\left.(2.14),(2.17),(2.32),(2.33),(2.44)\right)$, приходим к выводу, что

$$
\max _{-\sigma_{0} \leqslant t \leqslant T_{0}+\sigma_{0}}\left|x_{\varphi}(t, \varepsilon)-x_{0}(t)\right|=O(\varepsilon),
$$

где $x_{0}(t)$ - функция (2.9), а остаток равномерен по $\varphi \in S\left(\sigma_{0}, q_{1}, q_{2}\right)$.

Формулы (2.45), (2.46) свидетельствуют о том, что оператор (2.12) действительно определен на множестве $S\left(\sigma_{0}, q_{1}, q_{2}\right)$ и равномерно по $\varphi$

$$
\max _{-1-\sigma_{0} \leqslant t \leqslant-\sigma_{0}}\left|x_{\varphi}\left(t+T_{\varphi}, \varepsilon\right)-x_{0}(t)\right|=O(\varepsilon) .
$$

Что же касается требуемого включения $\Pi_{\varepsilon}\left(S\left(\sigma_{0}, q_{1}, q_{2}\right)\right) \subset S\left(\sigma_{0}, q_{1}, q_{2}\right)$, то в силу (2.47) оно будет заведомо выполняться при всех достаточно малых $\varepsilon>0$ при условии

$$
x_{0}(t) \in \widehat{S}\left(\sigma_{0}, q_{1}, q_{2}\right)
$$


где $\widehat{S}\left(\sigma_{0}, q_{1}, q_{2}\right)$ - множество функций, получающееся из $S\left(\sigma_{0}, q_{1}, q_{2}\right)$ при замене в (2.11) нестрогих неравенств строгими. Напомним, далее, что на параметр $\sigma_{0}$ нами уже наложено ограничение $(2.3)$, обеспечивающее свойства $x_{0}\left(-\sigma_{0}\right)=-\sigma_{0}$ и $x_{0}(t)<0$ при $-1-\sigma_{0} \leqslant t \leqslant-\sigma_{0}$. Поэтому справедливости включения $(2.48)$ добиваемся за счет имеющихся в запасе параметров $q_{1}, q_{2}$, предполагая, что

$$
q_{1}>-\min _{-1-\sigma_{0} \leqslant t \leqslant-\sigma_{0}} x_{0}(t), \quad 0<q_{2}<-\max _{-1-\sigma_{0} \leqslant t \leqslant-\sigma_{0}} x_{0}(t) .
$$

Итак, оператор $\Pi_{\varepsilon}$, очевидным образом являющийся компактным, при выполнении условий $(2.3),(2.15),(2.25),(2.49)$ на параметры $\sigma_{0}, q_{1}, q_{2}$ преобразует в себя замкнутое, ограниченное и выпуклое множество $S\left(\sigma_{0}, q_{1}, q_{2}\right)$. А отсюда в соответствии с известным принципом Шаудера заключаем, что этот оператор имеет в $S\left(\sigma_{0}, q_{1}, q_{2}\right)$ по крайней мере одну неподвижную точку $\varphi=\varphi_{*}(t, \varepsilon)$. Ясно также, что решение $x_{*}(t, \varepsilon)$ уравнения $(2.1)$ с начальной функцией $\varphi_{*}(t, \varepsilon)$, $-1-\sigma_{0} \leqslant t \leqslant-\sigma_{0}$, оказывается периодическим с периодом $T_{*}(\varepsilon)=\left.T_{\varphi}\right|_{\varphi=\varphi_{*}}$ и в силу (2.45)-(2.47) удовлетворяет требуемым свойствам (2.10).

2.3. Анализ свойств устойчивости. Перейдем теперь ко второй части обоснования теоремы 2.1, т. е. к доказательству единственности и устойчивости релаксационного цикла $x_{*}(t, \varepsilon)$ с нулевым приближением $(2.9)$. Из явной формулы (2.12) для оператора $\Pi_{\varepsilon}$ вытекает, что он непрерывно дифференцируем по $\varphi$, а его производная Фреше $\partial_{\varphi} \Pi_{\varepsilon}(\varphi)$ задается равенством

$$
\partial_{\varphi} \Pi_{\varepsilon}(\varphi) g_{0}=g\left(t+T_{\varphi}, \varepsilon\right)-l\left(g_{0}\right) \dot{x}_{\varphi}\left(t+T_{\varphi}, \varepsilon\right), \quad-1-\sigma_{0} \leqslant t \leqslant-\sigma_{0} .
$$

Здесь функция $g_{0}(t)$ представляет собой произвольный элемент пространства

$$
C_{0}=\left\{g_{0}(t) \in C\left[-1-\sigma_{0},-\sigma_{0}\right], g_{0}\left(-\sigma_{0}\right)=0\right\},
$$

через $g(t, \varepsilon),-\sigma_{0} \leqslant t \leqslant T_{\varphi}-\sigma_{0}$, обозначено решение линейного уравнения

$$
\dot{g}=A(t, \varepsilon) g(t-1), \quad A(t, \varepsilon)=\left.\frac{1}{\varepsilon}\left(f^{\prime}(\exp x) \exp x\right)\right|_{x=x_{\varphi}(t-1, \varepsilon) / \varepsilon}
$$

с начальной функцией $g_{0}(t),-1-\sigma_{0} \leqslant t \leqslant-\sigma_{0}$, а линейный функционал $l: C_{0} \rightarrow \mathbb{R}$ определен формулой

$$
l\left(g_{0}\right)=\frac{g\left(T_{\varphi}-\sigma_{0}, \varepsilon\right)}{\dot{x}_{\varphi}\left(T_{\varphi}-\sigma_{0}, \varepsilon\right)} .
$$

Из соотношений $(2.50),(2.52)$ следует, что проблема оценки нормы линейного оператора $\partial_{\varphi} \Pi_{\varepsilon}(\varphi)$ в пространстве $C_{0}$ с нормой $\left\|g_{0}\right\|=\max _{-1-\sigma_{0} \leqslant t \leqslant-\sigma_{0}}\left|g_{0}(t)\right|$ сводится к анализу введенного выше решения $g(t, \varepsilon)$ уравнения (2.51). Покажем, что для этого решения выполняется неравенство вида

$$
\max _{-\sigma_{0} \leqslant t \leqslant T_{\varphi}-\sigma_{0}}|g(t, \varepsilon)| \leqslant M \exp \left(-\frac{q}{\varepsilon}\right)\left\|g_{0}\right\|
$$

с некоторыми универсальными (не зависящими от $\varepsilon, \varphi, g_{0}$ ) положительными постоянными $M, q$. 
Обратимся сначала к отрезку $-\sigma_{0} \leqslant t \leqslant 1-\sigma_{0}$, на котором, в силу $(2.51)$, для $g(t, \varepsilon)$ имеем явную формулу

$$
g(t, \varepsilon)=\int_{-\sigma_{0}}^{t} A(s, \varepsilon) g_{0}(s-1) d s, \quad-\sigma_{0} \leqslant t \leqslant 1-\sigma_{0} .
$$

Напомним, далее, что при рассматриваемых $t$ решение $x_{\varphi}(t-1, \varepsilon)$ совпадает с функцией $\varphi(t-1)$, удовлетворяющей условиям (2.11). А отсюда и из свойств (1.6) функции $f(x)$ следует, что фигурирующий в $(2.51)$ коэффициент $A(t, \varepsilon)$ допускает оценку вида

$$
\max _{-\sigma_{0} \leqslant t \leqslant 1-\sigma_{0}}|A(t, \varepsilon)| \leqslant M \exp \left(-\frac{q}{\varepsilon}\right) .
$$

И наконец, учитывая (2.55) в (2.54), убеждаемся, что

$$
\max _{-\sigma_{0} \leqslant t \leqslant 1-\sigma_{0}}|g(t, \varepsilon)| \leqslant M \exp \left(-\frac{q}{\varepsilon}\right)\left\|g_{0}\right\| .
$$

Для распространения оценки (2.56) на оставшийся отрезок $\left[1-\sigma_{0}, T_{\varphi}-\sigma_{0}\right]$ изменения $t$ воспользуемся методом шагов. А именно, разобьем указанный промежуток времени на отрезки $\left[1-\sigma_{0}+k, 2-\sigma_{0}+k\right], k=0,1, \ldots, k_{0}$, и $\left[2-\sigma_{0}+k_{0}, T_{\varphi}-\sigma_{0}\right]$, где $k_{0}=\left\lfloor T_{\varphi}-2\right\rfloor,\lfloor *\rfloor$ - целая часть. Опираясь, далее, на равномерную по $\varphi \in S\left(\sigma_{0}, q_{1}, q_{2}\right)$ асимптотическую формулу

$$
\max _{1-\sigma_{0} \leqslant t \leqslant T_{\varphi}-\sigma_{0}}|A(t, \varepsilon)|=O\left(\frac{1}{\varepsilon}\right),
$$

замечаем, что из неравенства

$$
|g(t, \varepsilon)| \leqslant\left|g\left(1-\sigma_{0}+k, \varepsilon\right)\right|+\int_{1-\sigma_{0}+k}^{t}|A(s, \varepsilon)| \cdot|g(s-1, \varepsilon)| d s, \quad t \geqslant 1-\sigma_{0}+k,
$$

и из уже полученной оценки вида $(2.56)$ на $(k-1)$-м отрезке вытекает требуемая оценка на $k$-м отрезке изменения $t$.

Возвращаясь к оператору $\Pi_{\varepsilon}$ и учитывая в $(2.50),(2.52)$ установленное выше неравенство (2.53), приходим к выводу, что

$$
\sup _{\varphi \in S\left(\sigma_{0}, q_{1}, q_{2}\right)}\left\|\partial_{\varphi} \Pi_{\varepsilon}(\varphi)\right\|_{C_{0} \rightarrow C_{0}} \leqslant M \exp \left(-\frac{q}{\varepsilon}\right) .
$$

Остается добавить, что оценка (2.57) обеспечивает как сжимаемость оператора $\Pi_{\varepsilon}$ (а значит, единственность его неподвижной точки $\varphi=\varphi_{*}(t, \varepsilon)$ в множестве $\left.S\left(\sigma_{0}, q_{1}, q_{2}\right)\right)$, так и экспоненциальную орбитальную устойчивость соответствующего цикла $x_{*}(t, \varepsilon)$. Последнее обстоятельство обусловлено тем, что согласно (2.57) спектральный радиус оператора $\partial_{\varphi} \Pi_{\varepsilon}\left(\varphi_{*}\right)$ экспоненциально мал. Таким образом, все мультипликаторы $\mu$ цикла $x_{*}(t, \varepsilon)$, являющиеся (за исключением простого единичного) собственными значениями упомянутого оператора, лежат в круге $\{\mu \in \mathbb{C}:|\mu| \leqslant M \exp (-q / \varepsilon)\}$. Теорема 2.1 полностью доказана. 
В дополнение к установленной теореме отметим, что релаксационный цикл

$$
N_{*}(t, \lambda)=\left.\exp \left(\frac{x_{*}(t, \varepsilon)}{\varepsilon}\right)\right|_{\varepsilon=1 / \lambda}
$$

исходного уравнения (1.5) в силу (2.46) обладает требуемыми асимптотическими характеристиками (1.9), а для его периода $T_{*}(\lambda)$ справедлива вытекающая из (2.45) формула

$$
T_{*}(\lambda)=T_{0}+O(\exp (-q \lambda)), \quad q=\text { const }>0 .
$$

\section{3. Автоволновые режимы билокальной модели}

3.1. Основные теоремы. Изучение аттракторов диффузионной цепочки (1.10) начнем с ее частного случая

$$
\begin{aligned}
& \dot{N}_{1}=d\left(N_{2}-N_{1}\right)+\lambda f\left(N_{1}(t-1)\right) N_{1}, \\
& \dot{N}_{2}=d\left(N_{1}-N_{2}\right)+\lambda f\left(N_{2}(t-1)\right) N_{2},
\end{aligned}
$$

называемого билокальной моделью. Точнее говоря, последующий анализ будем проводить для системы

$$
\begin{aligned}
& \dot{x}_{1}=\varepsilon d\left(\exp \left(\frac{x_{2}-x_{1}}{\varepsilon}\right)-1\right)+f\left(\exp \left(\frac{x_{1}(t-1)}{\varepsilon}\right)\right), \\
& \dot{x}_{2}=\varepsilon d\left(\exp \left(\frac{x_{1}-x_{2}}{\varepsilon}\right)-1\right)+f\left(\exp \left(\frac{x_{2}(t-1)}{\varepsilon}\right)\right)
\end{aligned}
$$

получающейся из (3.1) при $N_{j}=\exp \left(x_{j} / \varepsilon\right), j=1,2, \varepsilon=1 / \lambda$.

Система (3.2) имеет, очевидно, однородный цикл $x_{1}=x_{2}=x_{*}(t, \varepsilon)$, где $x_{*}(t, \varepsilon)$ - релаксационный цикл уравнения (2.1), доставляемый теоремой 2.1. Ниже будет установлено, что, во-первых, этот цикл экспоненциально орбитально устойчив при любом фиксированном $d>0$ и при $\varepsilon \ll 1$; во-вторых, при надлежащем уменьшении параметра $d$ в билокальной модели (3.2) наряду с однородным циклом существуют и другие аттракторы. Таковыми являются два ее неоднородных цикла, переходящие друг в друга в результате замены

$$
\left(x_{1}, x_{2}\right) \rightarrow\left(x_{2}, x_{1}\right) .
$$

По ряду технических причин, которые прояснятся в последующем, отыскание автоволновых процессов системы (3.2) будем проводить при дополнительном условии

$$
a_{0}>1
$$

когда свойства релаксационного цикла модифицированного уравнения Хатчинсона (1.5) наиболее близки к свойствам цикла оригинального уравнения (1.1). Кроме того, для удобства дальнейшего асимптотического анализа перейдем в (3.2) к новым переменным $x=x_{1}, y=\left(x_{2}-x_{1}\right) / \varepsilon$. В результате интересующая нас система примет вид

$$
\begin{aligned}
& \dot{x}=\varepsilon d(\exp y-1)+F(x(t-1), \varepsilon), \\
& \dot{y}=-2 d \operatorname{sh} y+G(x(t-1), y(t-1), \varepsilon),
\end{aligned}
$$


где функция $F(x, \varepsilon)$ та же самая, что и в $(2.1)$, а $G(x, y, \varepsilon)$ задается формулой

$$
G(x, y, \varepsilon)=\frac{1}{\varepsilon}\left\{f\left(\exp \left(\frac{x}{\varepsilon}+y\right)\right)-f\left(\exp \left(\frac{x}{\varepsilon}\right)\right)\right\} .
$$

Зафиксируем постоянную $\sigma_{0}$, подчиненную требованиям $0<\sigma_{0}<a_{0}-1$, и рассмотрим банахово пространство $\mathscr{F}$ непрерывных при $-1-\sigma_{0} \leqslant t \leqslant-\sigma_{0}$ начальных вектор-функций $\varphi(t)=\left(\varphi_{1}(t), \varphi_{2}(t)\right)$ с нормой

$$
\|\varphi\|_{\mathscr{F}}=\max _{j}\left(\max _{-1-\sigma_{0} \leqslant t \leqslant-\sigma_{0}}\left|\varphi_{j}(t)\right|\right) .
$$

Всюду ниже нас будут интересовать решения системы (3.5) с начальными условиями из множества

$$
S=\left\{\varphi(t)=\left(\varphi_{1}(t), \varphi_{2}(t)\right): \varphi_{1} \in S_{1}, \varphi_{2} \in S_{2}\right\} \subset \mathscr{F} .
$$

Здесь через $S_{1}$ обозначена введенная при доказательстве теоремы 2.1 совокупность непрерывных функций $S\left(\sigma_{0}, q_{1}, q_{2}\right)$, параметры $q_{1}, q_{2}$ в которой удовлетворяют неравенствам (2.49), а в качестве $S_{2}$ взято произвольное замкнутое и ограниченное подмножество пространства $C\left[-1-\sigma_{0},-\sigma_{0}\right]$.

Формулировка строгих результатов об автоволновых режимах системы (3.5) требует некоторых подготовительных построений. В связи с этим введем в рассмотрение решение $\left(x_{\varphi}(t, \varepsilon), y_{\varphi}(t, \varepsilon)\right), t \geqslant-\sigma_{0}$, упомянутой системы, отвечающее произвольному начальному условию $\varphi(t) \in S$. Рассмотрим также второй положительный корень $t=T_{\varphi}$ уравнения $x_{\varphi}\left(t-\sigma_{0}, \varepsilon\right)=-\sigma_{0}$ (в случае, когда он существует) и на множестве (3.8) определим оператор $\Pi_{\varepsilon}: S \rightarrow \mathscr{F}$ посредством аналогичного (2.12) равенства

$$
\Pi_{\varepsilon}(\varphi)=\left(x_{\varphi}\left(t+T_{\varphi}, \varepsilon\right), y_{\varphi}\left(t+T_{\varphi}, \varepsilon\right)\right), \quad-1-\sigma_{0} \leqslant t \leqslant-\sigma_{0} .
$$

Помимо (3.9) нам потребуется еще оператор $\Pi_{0}: S \rightarrow \mathscr{F}$, который зададим формулой

$$
\Pi_{0}(\varphi)=\left.\left(x_{0}(t), y_{0}\left(t+T_{0}, z\right)\right)\right|_{z=\varphi_{2}\left(-\sigma_{0}\right)}, \quad-1-\sigma_{0} \leqslant t \leqslant-\sigma_{0} .
$$

Здесь, как и ранее, $T_{0}$ - величина (1.8), а $x_{0}(t)$ - периодическая функция (2.9). Что же касается компоненты $y_{0}(t, z), z \in \mathbb{R}$, то при $-\sigma_{0} \leqslant t \leqslant T_{0}-\sigma_{0}$ она является решением задачи Коши

$$
\begin{gathered}
\dot{y}=-2 d \operatorname{sh} y,\left.\quad y\right|_{t=-\sigma_{0}}=z, \\
y(1+0)=y(1-0)-\left(1+a_{0}\right) y(0), \\
y\left(t_{0}+1+0\right)=y\left(t_{0}+1-0\right)-\left(1+\frac{1}{a_{0}}\right) y\left(t_{0}\right) .
\end{gathered}
$$

Подчеркнем, что задача (3.11), (3.12) относится к так называемым системам с импульсным воздействием. Это означает, что в моменты времени $t=1$ и $t=t_{0}+1$, где $t_{0}$ - величина (2.7), ее решение $y_{0}(t, z)$ претерпевает конечные скачки, вычисляемые по правилам (3.12). Однако в силу неравенства (3.4) 
и оценки $\sigma_{0}<a_{0}-1$ функция $y_{0}\left(t+T_{0}, z\right)$ оказывается непрерывной на нужном отрезке $-1-\sigma_{0} \leqslant t \leqslant-\sigma_{0}$. Тем самым, условие (3.4) гарантирует выполнение требуемого включения $\Pi_{0}(\varphi) \in \mathscr{F}$ при всех $\varphi \in S$.

Завершая описание подготовительной части, рассмотрим производные Фреше $\partial_{\varphi} \Pi_{\varepsilon}(\varphi), \partial_{\varphi} \Pi_{0}(\varphi)$ операторов $(3.9)$, (3.10) по переменной $\varphi$. Проводя соответствующий подсчет, убеждаемся, что в данном случае эти производные представляют собой линейные операторы, действующие в пространстве

$$
\mathscr{F}_{0}=\left\{g_{0}(t)=\left(g_{1,0}(t), g_{2,0}(t)\right) \in \mathscr{F}: g_{1,0}\left(-\sigma_{0}\right)=0\right\}
$$

с нормой (3.7), а результаты их применения к произвольному элементу $g_{0}(t) \in \mathscr{F}_{0}$ задаются соответственно равенствами

$$
\begin{aligned}
& \partial_{\varphi} \Pi_{\varepsilon}(\varphi) g_{0}=\left(g_{1}\left(t+T_{\varphi}, \varepsilon\right), g_{2}\left(t+T_{\varphi}, \varepsilon\right)\right) \\
& \quad-l\left(g_{0}\right)\left(\dot{x}_{\varphi}\left(t+T_{\varphi}, \varepsilon\right), \dot{y}_{\varphi}\left(t+T_{\varphi}, \varepsilon\right)\right), \quad-1-\sigma_{0} \leqslant t \leqslant-\sigma_{0} ; \\
& \partial_{\varphi} \Pi_{0}(\varphi) g_{0}=\left(0,\left.\frac{\partial y_{0}}{\partial z}\left(t+T_{0}, z\right)\right|_{z=\varphi_{2}\left(-\sigma_{0}\right)}\right) g_{2,0}\left(-\sigma_{0}\right), \quad-1-\sigma_{0} \leqslant t \leqslant-\sigma_{0} .
\end{aligned}
$$

Здесь $g(t, \varepsilon)=\left(g_{1}(t, \varepsilon), g_{2}(t, \varepsilon)\right),-\sigma_{0} \leqslant t \leqslant T_{\varphi}-\sigma_{0},-$ решение линейной системы

$$
\begin{aligned}
& \dot{g}_{1}=a_{11}(t, \varepsilon) g_{1}(t-1)+a_{12}(t, \varepsilon) g_{2}, \\
& \dot{g}_{2}=a_{21}(t, \varepsilon) g_{1}(t-1)+a_{22}(t, \varepsilon) g_{2}+a_{23}(t, \varepsilon) g_{2}(t-1)
\end{aligned}
$$

с коэффициентами

$$
\begin{array}{cc}
a_{11}=\left.\frac{\partial F}{\partial x}(x, \varepsilon)\right|_{x=x_{\varphi}(t-1, \varepsilon)}, & a_{12}=\varepsilon d \exp \left(y_{\varphi}(t, \varepsilon)\right), \\
a_{21}=\left.\frac{\partial G}{\partial x}(x, y, \varepsilon)\right|_{x=x_{\varphi}(t-1, \varepsilon), y=y_{\varphi}(t-1, \varepsilon)}, & a_{22}=-2 d \operatorname{ch}\left(y_{\varphi}(t, \varepsilon)\right), \\
a_{23}=\left.\frac{\partial G}{\partial y}(x, y, \varepsilon)\right|_{x=x_{\varphi}(t-1, \varepsilon), y=y_{\varphi}(t-1, \varepsilon)}
\end{array}
$$

и начальной функцией $g_{0}(t)$ из пространства $(3.13)$, а функционал $l: \mathscr{F}_{0} \rightarrow \mathbb{R}$ определен аналогичным (2.52) соотношением

$$
l\left(g_{0}\right)=\frac{g_{1}\left(T_{\varphi}-\sigma_{0}, \varepsilon\right)}{\dot{x}_{\varphi}\left(T_{\varphi}-\sigma_{0}, \varepsilon\right)} .
$$

Зададимся естественно возникающим вопросом о связи между операторами (3.9) и (3.10). Ответ на него содержится в следующем утверждении.

Теорема 3.1 (о $C^{1}$-сходимости). Пусть выполнено условие (3.4) и множество $S$ выбрано описанным выше способом. Тогда найдется такое достаточно малое $\varepsilon_{0}=\varepsilon_{0}(S)>0$, что при всех $0<\varepsilon \leqslant \varepsilon_{0}$ оператор $\Pi_{\varepsilon}$ определен на $S$ и удовлетворяет предельным равенствам

$$
\lim _{\varepsilon \rightarrow 0} \sup _{\varphi \in S}\left\|\Pi_{\varepsilon}(\varphi)-\Pi_{0}(\varphi)\right\|_{\mathscr{F}}=0, \quad \lim _{\varepsilon \rightarrow 0} \sup _{\varphi \in S}\left\|\partial_{\varphi} \Pi_{\varepsilon}(\varphi)-\partial_{\varphi} \Pi_{0}(\varphi)\right\|_{\mathscr{F}_{0} \rightarrow \mathscr{F}_{0}}=0 .
$$


Обоснованию данной теоремы посвятим отдельный пункт. Здесь же остановимся на одном важном следствии из $C^{1}$-сходимости, касающемся существования и устойчивости периодических решений системы (3.5).

Заметим, что в силу (3.11), (3.12) предельный оператор (3.10) является надстройкой над соответствующим одномерным отображением

$$
\left.z \rightarrow \Phi(z) \stackrel{\text { def }}{=} y_{0}(t, z)\right|_{t=T_{0}-\sigma_{0}}
$$

где $z=\varphi_{2}\left(-\sigma_{0}\right)$. Действительно, любой неподвижной точке $z=z_{*}$ этого отображения соответствует неподвижная точка

$\varphi_{*}(t)=\left(\varphi_{1}^{*}(t), \varphi_{2}^{*}(t)\right): \quad \varphi_{1}^{*}(t)=x_{0}(t), \quad \varphi_{2}^{*}(t)=y_{0}\left(t+T_{0}, z_{*}\right), \quad-1-\sigma_{0} \leqslant t \leqslant-\sigma_{0}$,

оператора $\Pi_{0}$ (при условии, конечно, что $\varphi_{2}^{*}(t)$ принадлежит множеству $S_{2}$ из (3.8)). Последнее же требование не является ограничением, поскольку, как уже говорилось выше, $S_{2}$ можно считать произвольным замкнутым и ограниченным подмножеством пространства $C\left[-1-\sigma_{0},-\sigma_{0}\right]$.

Верно и обратное утверждение: если $\varphi_{*}(t)=\left(\varphi_{1}^{*}(t), \varphi_{2}^{*}(t)\right) \in S$ есть неподвижная точка оператора $\Pi_{0}$, то с необходимостью $\varphi_{1}^{*}(t)=x_{0}(t)$, а величина $z_{*}=\varphi_{2}^{*}\left(-\sigma_{0}\right)$ такова, что $\Phi\left(z_{*}\right)=z_{*}$. Кроме того, в силу (3.15) спектр линейного оператора $\partial_{\varphi} \Pi_{0}\left(\varphi_{*}\right)$ состоит из двух точек: собственного значения $\mu=0$ бесконечной кратности и простого собственного значения $\mu=\Phi^{\prime}\left(z_{*}\right)$.

Суммируя изложенные факты, приходим к выводу, что справедлив следующий результат.

Теорема 3.2 (о соответствии). Каждой неподвижной точке $z=z_{*}$, $\left|\Phi^{\prime}\left(z_{*}\right)\right| \neq 1$ отображения (3.22) соответствует релаксационный цикл системы (3.5), существующий при всех достаточно мальх $\varepsilon>0$ и являющийся экспоненциально орбитально устойчивым (неустойчивым) в случае $\left|\Phi^{\prime}\left(z_{*}\right)\right|<1(>1)$.

ДокАзАТЕЛЬСтво. Обратимся сначала к вопросу о существовании релаксационного цикла. В связи с этим обозначим через $\varphi_{*}(t) \in S$ неподвижную точку оператора (3.10), отвечающую неподвижной точке $z=z_{*}$ отображения (3.22). Рассмотрим, далее, уравнение

$$
\Pi_{\varepsilon}(\varphi)-\varphi=0
$$

и заметим, что в силу предельных равенств (3.21) и отмеченных выше спектральных свойств оператора $\partial_{\varphi} \Pi_{0}\left(\varphi_{*}\right)$ к данному уравнению в точке $(\varphi, \varepsilon)=$ $\left(\varphi_{*}(t), 0\right)$ пространства $\mathscr{F} \times \mathbb{R}$ применима теорема о неявном отображении по переменной $\varphi$. Таким образом, из (3.23) однозначно определяется неподвижная точка

$$
\varphi=\varphi_{*}^{\varepsilon}(t) \in S, \quad \lim _{\varepsilon \rightarrow 0}\left\|\varphi_{*}^{\varepsilon}(t)-\varphi_{*}(t)\right\|_{\mathscr{F}}=0,
$$

оператора (3.9), а отвечающее ей решение $\left.\left(x_{\varphi}(t, \varepsilon), y_{\varphi}(t, \varepsilon)\right)\right|_{\varphi=\varphi_{*}^{\varepsilon}}$ системы $(3.5)$ будет, очевидно, периодическим с периодом $T=\left.T_{\varphi}\right|_{\varphi=\varphi_{*}^{\varepsilon}}$.

Перейдем теперь к вопросу об устойчивости найденного периодического решения. Из проделанных выше построений следует, что все его мультипликаторы (за исключением простого единичного) являются собственными значениями 
оператора $\partial_{\varphi} \Pi_{\varepsilon}\left(\varphi_{*}^{\varepsilon}\right)$. Последний же в силу вытекающего из (3.21) равенства

$$
\lim _{\varepsilon \rightarrow 0}\left\|\partial_{\varphi} \Pi_{\varepsilon}\left(\varphi_{*}^{\varepsilon}\right)-\partial_{\varphi} \Pi_{0}\left(\varphi_{*}\right)\right\|_{\mathscr{F}_{0} \rightarrow \mathscr{F}_{0}}=0
$$

имеет одно простое собственное значение, асимптотически близкое к $\Phi^{\prime}\left(z_{*}\right)$, а остальной его спектр лежит в круге $\left\{\mu \in \mathbb{C}:|\mu| \leqslant r_{0}\right\}$ асимптотически малого по $\varepsilon$ радиуса $r_{0}=r_{0}(\varepsilon)$. Таким образом, свойства устойчивости рассматриваемого цикла совпадают с аналогичными свойствами неподвижной точки $z=z_{*}$ отображения (3.22). Теорема 3.2 доказана.

Установленная теорема сводит интересующую нас проблему автоволновых процессов системы (3.5) к поиску неподвижных точек одномерного отображения (3.22). Вопрос же о количестве и устойчивости последних будет изучен отдельно в п. 3.3 .

3.2. Обоснование $C^{1}$-сходимости. Доказательство соотношений (3.21) базируется на построении равномерной по $\varphi \in S$ асимптотики введенного выше решения $\left(x_{\varphi}(t, \varepsilon), y_{\varphi}(t, \varepsilon)\right)$ системы $(3.5)$. Как и в предыдущем разделе, соответствующий анализ начнем с отрезка $-\sigma_{0} \leqslant t \leqslant 1-\sigma_{0}$, на котором в силу условий (2.11), наложенных на $\varphi_{1}(t)$, и свойств (1.6) функции $f(x)$ справедливы равномерные по $\varphi \in S$ асимптотические представления

$$
\begin{gathered}
F\left(\varphi_{1}(t-1), \varepsilon\right)=1+O\left(\exp \left(-\frac{q}{\varepsilon}\right)\right), \\
G\left(\varphi_{1}(t-1), \varphi_{2}(t-1), \varepsilon\right)=O\left(\exp \left(-\frac{q}{\varepsilon}\right)\right), \\
q=\text { const }>0 .
\end{gathered}
$$

Учитывая эти равенства, из (3.5) выводим, что при рассматриваемых $t$ с экспоненциальной по $\varepsilon$ точностью

$$
\left(x_{\varphi}(t, \varepsilon), y_{\varphi}(t, \varepsilon)\right)=(\tilde{x}(t, z, \varepsilon), \tilde{y}(t, z, \varepsilon)),
$$

где функции $\tilde{x}, \tilde{y}$, определяемые из задачи Коши

$$
\dot{x}=\varepsilon d(\exp y-1)+1, \quad \dot{y}=-2 d \operatorname{sh} y,\left.\quad x\right|_{t=-\sigma_{0}}=-\sigma_{0},\left.\quad y\right|_{t=-\sigma_{0}}=z,
$$

имеют вид

$$
\tilde{x}=t+\varepsilon d \int_{-\sigma_{0}}^{t}\left[\exp \left(y_{0}(s, z)\right)-1\right] d s, \quad \tilde{y}=y_{0}(t, z) .
$$

Добавим еще, что здесь и ниже параметр $z$ задан равенством $z=\varphi_{2}\left(-\sigma_{0}\right)$, а значит, меняется на некотором компактном подмножестве числовой оси.

На следующем этапе фиксируем постоянную $\delta \in(0,1)$ и рассмотрим отрезок $1-\sigma_{0} \leqslant t \leqslant 1-\varepsilon^{\delta}$. Разбивая его, если это необходимо, на части длины не более единицы и применяя метод шагов (см. комментарий после формулы (2.32)), убеждаемся в пригодности равенств (3.24) вплоть до точки $t=1-\varepsilon^{\delta}$. Однако в отличие от предыдущего случая здесь в силу оценки $x_{\varphi}(t-1, \varepsilon) \leqslant-M \varepsilon^{\delta}$, 
$M=$ const $>0$, имеющей место при значениях $t$, близких к $t=1-\varepsilon^{\delta}$, указанные равенства выполняются с точностью до $O\left(\exp \left(-q / \varepsilon^{1-\delta}\right)\right), q=$ const $>0$.

Обратимся, далее, к отрезку $1-\varepsilon^{\delta} \leqslant t \leqslant 1+\varepsilon^{\delta}$. Нетрудно увидеть, что на этом промежутке времени интересующее нас решение $\left(x_{\varphi}(t, \varepsilon), y_{\varphi}(t, \varepsilon)\right)$ системы (3.5) совпадает (с точностью до величин порядка $\exp \left(-q / \varepsilon^{1-\delta}\right), q=$ const $>0)$ с решением задачи Коши

$$
\begin{gathered}
\dot{x}=\varepsilon d(\exp y-1)+F_{z}(t, \varepsilon), \quad \dot{y}=-2 d \operatorname{sh} y+G_{z}(t, \varepsilon), \\
\left.x\right|_{t=1-\varepsilon^{\delta}}=x_{z}(\varepsilon),\left.\quad y\right|_{t=1-\varepsilon^{\delta}}=y_{z}(\varepsilon),
\end{gathered}
$$

где

$$
\begin{gathered}
F_{z}=F(\tilde{x}(t-1, z, \varepsilon), \varepsilon), \quad G_{z}=G(\tilde{x}(t-1, z, \varepsilon), \tilde{y}(t-1, z, \varepsilon), \varepsilon), \\
x_{z}(\varepsilon)=\tilde{x}\left(1-\varepsilon^{\delta}, z, \varepsilon\right), \quad y_{z}(\varepsilon)=\tilde{y}\left(1-\varepsilon^{\delta}, z, \varepsilon\right),
\end{gathered}
$$

а $\tilde{x}, \tilde{y}$ - функции из (3.24).

Асимптотический анализ задачи (3.27) будем проводить в переменных $v_{1}=$ $(x-1) / \varepsilon, v_{2}=y, \tau=(t-1) / \varepsilon$, после перехода к которым она принимает вид

$$
\begin{gathered}
\frac{d v_{1}}{d \tau}=\varepsilon d\left(\exp v_{2}-1\right)+H_{1}(\tau, z, \varepsilon), \quad \frac{d v_{2}}{d \tau}=-2 \varepsilon d \operatorname{sh} v_{2}+H_{2}(\tau, z, \varepsilon), \\
\left.v_{1}\right|_{\tau=-\varepsilon^{\delta-1}}=\bar{v}_{1}(z, \varepsilon),\left.\quad v_{2}\right|_{\tau=-\varepsilon^{\delta-1}}=\bar{v}_{2}(z, \varepsilon) .
\end{gathered}
$$

Здесь $\bar{v}_{1}=\left(x_{z}(\varepsilon)-1\right) / \varepsilon, \bar{v}_{2}=y_{z}(\varepsilon)$, переменная $\tau$ меняется на асимптотически большом отрезке $J(\varepsilon)=\left[-\varepsilon^{\delta-1}, \varepsilon^{\delta-1}\right]$, а функции $H_{1}, H_{2}$ задаются следующими равенствами:

$$
\begin{aligned}
H_{1} & =f(x) \quad \text { при } x=\exp \left(\frac{\tilde{x}(\varepsilon \tau, z, \varepsilon)}{\varepsilon}\right) \\
H_{1}+H_{2} & =f(x) \quad \text { при } x=\exp \left(\frac{\tilde{x}(\varepsilon \tau, z, \varepsilon)}{\varepsilon}+\tilde{y}(\varepsilon \tau, z, \varepsilon)\right) .
\end{aligned}
$$

Обозначим через $\left(v_{1}(\tau, z, \varepsilon), v_{2}(\tau, z, \varepsilon)\right), \tau \in J(\varepsilon)$, решение задачи (3.29). Для отыскания главных членов его асимптотики положим в уравнениях из (3.29) $\varepsilon=0$ и учтем формулы (3.26), (3.30). В результате приходим к системе

$$
\frac{d v_{1}}{d \tau}=f\left(\exp \left(\tau+\varkappa_{1}\right)\right), \quad \frac{d v_{2}}{d \tau}=f\left(\exp \left(\tau+\varkappa_{2}\right)\right)-f\left(\exp \left(\tau+\varkappa_{1}\right)\right),
$$

где функции $\varkappa_{j}=\varkappa_{j}(z), j=1,2$, имеют вид

$$
\varkappa_{1}=d \int_{-\sigma_{0}}^{0}\left[\exp \left(y_{0}(s, z)\right)-1\right] d s, \quad \varkappa_{2}=\varkappa_{1}+y_{0}(0, z) .
$$

Нетрудно проверить, что система (3.31) допускает решение $\left(v_{1}^{0}(\tau, z), v_{2}^{0}(\tau, z)\right)$ с компонентами

$$
v_{1}^{0}=v\left(\tau+\varkappa_{1}\right)+\varkappa_{3}, \quad v_{2}^{0}=v\left(\tau+\varkappa_{2}\right)-v\left(\tau+\varkappa_{1}\right)+\varkappa_{4},
$$


где $v(\tau)$ - функция (2.18), а $\varkappa_{3}(z), \varkappa_{4}(z)$ определяются аналогичными (3.32) равенствами

$$
\varkappa_{3}=d \int_{0}^{1}\left[\exp \left(y_{0}(s, z)\right)-1\right] d s, \quad \varkappa_{4}=y_{0}(1-0, z)-y_{0}(0, z) .
$$

Как оказывается, именно это решение и является искомым. Точнее говоря, справедливо следующее утверждение.

Лемма 3.1. Равномерно по $\tau \in J(\varepsilon)$ и по параметру z выполняются асимптотические равенства

$$
v_{j}(\tau, z, \varepsilon)=v_{j}^{0}(\tau, z)+O\left(\varepsilon^{\delta}\right), \quad \frac{\partial v_{j}}{\partial z}(\tau, z, \varepsilon)=\frac{\partial v_{j}^{0}}{\partial z}(\tau, z)+O\left(\varepsilon^{\delta}\right), \quad j=1,2 .
$$

ДокАЗАТЕЛЬство. Подставляя в (3.29) соотношения $v_{j}=v_{j}^{0}+h_{j}, j=1,2$, для $h_{1}, h_{2}$ приходим к задаче Коши

$$
\begin{aligned}
\frac{d h_{1}}{d \tau}=\varepsilon d & \left(\exp \left(v_{2}^{0}(\tau, z)+h_{2}\right)-1\right) \\
& +H_{1}(\tau, z, \varepsilon)-H_{1}(\tau, z, 0),\left.\quad h_{1}\right|_{\tau=-\varepsilon^{\delta-1}}=\omega_{1}(z, \varepsilon), \\
\frac{d h_{2}}{d \tau}=- & 2 \varepsilon d \operatorname{sh}\left(v_{2}^{0}(\tau, z)+h_{2}\right) \\
& +H_{2}(\tau, z, \varepsilon)-H_{2}(\tau, z, 0),\left.\quad h_{2}\right|_{\tau=-\varepsilon^{\delta-1}}=\omega_{2}(z, \varepsilon),
\end{aligned}
$$

где

$$
\omega_{j}(z, \varepsilon)=\bar{v}_{j}(z, \varepsilon)-\left.v_{j}^{0}(\tau, z)\right|_{\tau=-\varepsilon^{\delta-1}}, \quad j=1,2 .
$$

Далее, полагая $h_{3}=\partial h_{1} / \partial z, h_{4}=\partial h_{2} / \partial z$, дополним (3.36) соответствующими уравнениями

$$
\begin{aligned}
& \frac{d h_{3}}{d \tau}=\varepsilon d \exp \left(v_{2}^{0}(\tau, z)+h_{2}\right)\left(\frac{\partial v_{2}^{0}}{\partial z}+h_{4}\right)+\frac{\partial H_{1}}{\partial z}(\tau, z, \varepsilon)-\frac{\partial H_{1}}{\partial z}(\tau, z, 0), \\
& \frac{d h_{4}}{d \tau}=-2 \varepsilon d \operatorname{ch}\left(v_{2}^{0}(\tau, z)+h_{2}\right)\left(\frac{\partial v_{2}^{0}}{\partial z}+h_{4}\right)+\frac{\partial H_{2}}{\partial z}(\tau, z, \varepsilon)-\frac{\partial H_{2}}{\partial z}(\tau, z, 0)
\end{aligned}
$$

и начальными условиями

$$
\left.h_{3}\right|_{\tau=-\varepsilon^{\delta-1}}=\frac{\partial \omega_{1}}{\partial z}(z, \varepsilon),\left.\quad h_{4}\right|_{\tau=-\varepsilon^{\delta-1}}=\frac{\partial \omega_{2}}{\partial z}(z, \varepsilon) .
$$

И наконец, перейдем от получившейся задачи Коши (3.36)-(3.39) к эквивалентной системе интегральных уравнений

$$
\begin{array}{ll}
h_{1}=\omega_{1}+\int_{-\varepsilon^{\delta-1}}^{\tau} \Psi_{1}\left(s, z, \varepsilon, h_{2}\right) d s, & h_{2}=\omega_{2}+\int_{-\varepsilon^{\delta-1}}^{\tau} \Psi_{2}\left(s, z, \varepsilon, h_{2}\right) d s, \\
h_{3}=\frac{\partial \omega_{1}}{\partial z}+\int_{-\varepsilon^{\delta-1}}^{\tau} \Psi_{3}\left(s, z, \varepsilon, h_{2}, h_{4}\right) d s, & h_{4}=\frac{\partial \omega_{2}}{\partial z}+\int_{-\varepsilon^{\delta-1}}^{\tau} \Psi_{4}\left(s, z, \varepsilon, h_{2}, h_{4}\right) d s,
\end{array}
$$

где для краткости через $\Psi_{j}, j=1, \ldots, 4$, обозначены правые части соответствующих дифференциальных уравнений из (3.36), (3.38). 
Остановимся на некоторых свойствах функций $\omega_{j}(z, \varepsilon), H_{j}(\tau, z, \varepsilon), j=1,2$, и $v_{2}^{0}(\tau, z)$, необходимых для анализа системы (3.40). Учитывая в (3.37) явный вид начальных условий $\bar{v}_{j}(z, \varepsilon), j=1,2$, из (3.29) и опираясь на формулы (3.32)-(3.34), (2.18), (2.22), приходим к выводу, что равномерно по $z$

$$
\omega_{j}(z, \varepsilon)=O\left(\varepsilon^{\delta}\right), \quad \frac{\partial \omega_{j}}{\partial z}(z, \varepsilon)=O\left(\varepsilon^{\delta}\right), \quad j=1,2 .
$$

Перейдем теперь к функциям $H_{1}, H_{2}$. Из явных формул (3.30) и оценки (2.23) вытекают неравенства вида

$$
\left|H_{j}(\tau, z, \varepsilon)-H_{j}(\tau, z, 0)\right| \leqslant M \varepsilon^{\delta} \exp (-|\tau|), \quad \tau \in J(\varepsilon), \quad j=1,2 ;
$$

здесь и ниже одной и той же буквой $M$ обозначаются различные универсальные (не зависящие от $\varepsilon, \tau, z$ ) положительные постоянные, точные значения которых несущественны.

Обоснование аналогичных (3.42) оценок для $\partial H_{j} / \partial z, j=1,2$, базируется на асимптотическом разложении из (1.6). Дифференцируя его два раза подряд, последовательно убеждаемся, что

$$
\begin{aligned}
&\left|f^{\prime}(x)+x f^{\prime \prime}(x)\right| \leqslant \frac{M}{1+x^{2}} \quad \forall x \in \mathbb{R}_{+}, \\
&\left|x_{1} f^{\prime}\left(x_{1}\right)-x_{2} f^{\prime}\left(x_{2}\right)\right| \leqslant \frac{M}{1+\min \left(x_{1}^{2}, x_{2}^{2}\right)}\left|x_{1}-x_{2}\right| \quad \forall x_{1}, x_{2} \in \mathbb{R}_{+} .
\end{aligned}
$$

Применяя затем второе неравенство (3.43) к формулам, получающимся из соотношений (3.30) после дифференцирования по $z$, приходим к выводу, что

$$
\left|\frac{\partial H_{j}}{\partial z}(\tau, z, \varepsilon)-\frac{\partial H_{j}}{\partial z}(\tau, z, 0)\right| \leqslant M \varepsilon^{\delta} \exp (-|\tau|), \quad \tau \in J(\varepsilon), \quad j=1,2 .
$$

Обратим внимание, что в правые части уравнений из (3.36), (3.38) входят функции $v_{2}^{0}$ и $\partial v_{2}^{0} / \partial z$. А так как отрезок $J(\varepsilon)$, на котором рассматривается система (3.40), асимптотически велик, то в дальнейшем нам потребуется информация о поведении этих функций при $\tau \rightarrow \pm \infty$. Для ее получения учтем в (3.33) известные асимптотические формулы (2.22), (2.27). В результате имеем равномерные по $z$ предельные равенства

$$
\begin{array}{cl}
v_{2}^{0}=y_{0}(1-0, z)+O(\exp \tau), & \frac{\partial v_{2}^{0}}{\partial z}=\frac{\partial y_{0}}{\partial z}(1-0, z)+O(\exp \tau), \quad \tau \rightarrow-\infty \\
v_{2}^{0}=y_{0}(1+0, z)+O(\exp (-\tau)), & \frac{\partial v_{2}^{0}}{\partial z}=\frac{\partial y_{0}}{\partial z}(1+0, z)+O(\exp (-\tau)), \quad \tau \rightarrow+\infty,
\end{array}
$$

где $y_{0}(1+0, z)$ и $y_{0}(1-0, z)$ связаны требуемым соотношением из $(3.12)$. Добавим еще, что свойства (3.45) гарантируют равномерную по $\tau, z$ ограниченность указанных функций.

Приступим непосредственно к исследованию системы (3.40). В связи с этим обозначим через $V(h), h=\left(h_{1}, h_{2}, h_{3}, h_{4}\right)$, оператор, порожденный ее правыми частями в пространстве $C\left(J(\varepsilon) ; \mathbb{R}^{4}\right)$ с нормой $\|h\|=\max _{j}\left(\max _{\tau \in J(\varepsilon)}\left|h_{j}\right|\right)$. Объединяя свойства (3.41) с оценками (3.42), (3.44) и используя факт ограниченности по $\tau, z$ функций $v_{2}^{0}, \partial v_{2}^{0} / \partial z$, убеждаемся, что данный оператор преобразует 
в себя замкнутый шар пространства $C\left(J(\varepsilon) ; \mathbb{R}^{4}\right)$ с центром в нуле и радиусом вида $r=M \varepsilon^{\delta}$ и оказывается сжимающим (с константой сжатия вида $\left.M \varepsilon^{\delta}\right)$. Таким образом, он имеет в упомянутом шаре единственную неподвижную точку, компоненты $h_{j}(\tau, z, \varepsilon), j=1, \ldots, 4$, которой являются одновременно и компонентами решения задачи Коши (3.36)-(3.39). Лемма 3.1 доказана.

Возвращаясь к исходному решению $\left(x_{\varphi}(t, \varepsilon), y_{\varphi}(t, \varepsilon)\right)$, отметим, что в силу (3.35) для его компонент равномерно по $t \in\left[1-\varepsilon^{\delta}, 1+\varepsilon^{\delta}\right], \varphi \in S$ справедливы асимптотические представления

$x_{\varphi}(t, \varepsilon)=1+\left.\varepsilon v_{1}^{0}(\tau, z)\right|_{\tau=(t-1) / \varepsilon}+O\left(\varepsilon^{1+\delta}\right), \quad y_{\varphi}(t, \varepsilon)=\left.v_{2}^{0}(\tau, z)\right|_{\tau=(t-1) / \varepsilon}+O\left(\varepsilon^{\delta}\right)$.

Из этих формул, в частности, следует, что на рассматриваемом асимптотически малом промежутке изменения $t$ компонента $y_{\varphi}(t, \varepsilon)$ меняется существенно. А именно, из $(3.45),(3.46)$ вытекает, что $y_{\varphi}\left(1-\varepsilon^{\delta}, \varepsilon\right) \rightarrow y_{0}(1-0, z)$, $y_{\varphi}\left(1+\varepsilon^{\delta}, \varepsilon\right) \rightarrow y_{0}(1+0, z)$ при $\varepsilon \rightarrow 0$. Тем самым, придерживаясь принятой в теории релаксационных колебаний терминологии, данный отрезок будем называть участком быстрых движений.

Установленные выше равенства (3.24), (3.46) гарантируют выполнение на очередном отрезке $1+\varepsilon^{\delta} \leqslant t \leqslant 2+\varepsilon^{\delta}$ оценки вида $x_{\varphi}(t-1, \varepsilon) \geqslant M \varepsilon^{\delta}, M=$ const $>0$. А отсюда и из известных свойств функции $f(x)($ см. (1.6)) заключаем, что при указанных $t$ после отбрасывания добавок, обладающих равномерно по $\varphi \in S$ порядком малости $\exp \left(-q / \varepsilon^{1-\delta}\right), q=$ const $>0$, для $x_{\varphi}(t, \varepsilon), y_{\varphi}(t, \varepsilon)$ получаются аналогичные (3.24) формулы

$$
x_{\varphi}(t, \varepsilon)=\tilde{x}(t, z, \varepsilon), \quad y_{\varphi}(t, \varepsilon)=\tilde{y}(t, z, \varepsilon),
$$

где теперь $(\tilde{x}, \tilde{y})$ - решение аналогичной (3.25) задачи Коши

$$
\begin{gathered}
\dot{x}=\varepsilon d(\exp y-1)-a_{0}, \quad \dot{y}=-2 d \operatorname{sh} y, \\
\left.x\right|_{t=1+\varepsilon^{\delta}}=1+\left.\varepsilon v_{1}(\tau, z, \varepsilon)\right|_{\tau=\varepsilon^{\delta-1}},\left.\quad y\right|_{t=1+\varepsilon^{\delta}}=\left.v_{2}(\tau, z, \varepsilon)\right|_{\tau=\varepsilon^{\delta-1}} .
\end{gathered}
$$

Остановимся на асимптотических свойствах правых частей из (3.47). Учитывая в (3.48) асимптотические представления (3.35) и характер поведения при $\tau \rightarrow+\infty$ функций (3.33) (см. (2.27), (3.45)), после несложных преобразований приходим к выводу, что равномерно по $t, z$

$$
\begin{gathered}
\tilde{x}=1-a_{0}(t-1)+\varepsilon d \int_{1}^{t}\left[\exp \left(y_{0}(s, z)\right)-1\right] d s+\varepsilon c(z)+O\left(\varepsilon^{1+\delta}\right), \\
\frac{\partial \tilde{x}}{\partial z}=\frac{\partial}{\partial z}\left(\varepsilon d \int_{1}^{t}\left[\exp \left(y_{0}(s, z)\right)-1\right] d s+\varepsilon c(z)\right)+O\left(\varepsilon^{1+\delta}\right) ; \\
\tilde{y}=y_{0}(t, z)+O\left(\varepsilon^{\delta}\right), \quad \frac{\partial \tilde{y}}{\partial z}=\frac{\partial y_{0}}{\partial z}(t, z)+O\left(\varepsilon^{\delta}\right),
\end{gathered}
$$

где

$$
c(z)=d \int_{-\sigma_{0}}^{1}\left[\exp \left(y_{0}(s, z)\right)-1\right] d s-\left(1+a_{0}\right) d \int_{-\sigma_{0}}^{0}\left[\exp \left(y_{0}(s, z)\right)-1\right] d s+c_{0},
$$

a $c_{0}-$ постоянная (2.28). 
Необходимо отметить, что равенства (3.47), установленные нами при $t \in$ $\left[1+\varepsilon^{\delta}, 2+\varepsilon^{\delta}\right]$, на самом деле сохраняются (вместе со свойствами (3.49)-(3.51)) на более широком промежутке $1+\varepsilon^{\delta} \leqslant t \leqslant t_{0}+1-\varepsilon^{\delta}$. Связано это с тем, что при указанных $t$ остается в силе оценка вида $x_{\varphi}(t-1, \varepsilon) \geqslant M \varepsilon^{\delta}, M=$ const $>0$, справедливость которой проверяется методом шагов.

Последующий анализ вполне аналогичен описанному выше. Действительно, на очередном участке быстрых движений, т. е. при $t_{0}+1-\varepsilon^{\delta} \leqslant t \leqslant t_{0}+1+\varepsilon^{\delta}$, с точностью до $O\left(\exp \left(-q / \varepsilon^{1-\delta}\right)\right), q=$ const $>0$, pешение $\left(x_{\varphi}(t, \varepsilon), y_{\varphi}(t, \varepsilon)\right)$ совпадает с решением задачи Коши, получающейся из (3.27), (3.28) в результате замены начального момента времени $t=1-\varepsilon^{\delta}$ на $t=t_{0}+1-\varepsilon^{\delta}$, а функций $\tilde{x}, \tilde{y}-$ на соответствующие функции из (3.47). Далее, в переменных $w_{1}=\left(x+a_{0}\right) / \varepsilon$, $w_{2}=y, \tau=\left(t-t_{0}-1\right) / \varepsilon$ упомянутая задача принимает аналогичный $(3.29)$ вид

$$
\begin{gathered}
\frac{d w_{1}}{d \tau}=\varepsilon d\left(\exp w_{2}-1\right)+H_{1}(\tau, z, \varepsilon), \quad \frac{d w_{2}}{d \tau}=-2 \varepsilon d \operatorname{sh} w_{2}+H_{2}(\tau, z, \varepsilon), \\
\left.w_{1}\right|_{\tau=-\varepsilon^{\delta-1}}=\bar{w}_{1}(z, \varepsilon),\left.\quad w_{2}\right|_{\tau=-\varepsilon^{\delta-1}}=\bar{w}_{2}(z, \varepsilon) .
\end{gathered}
$$

Здесь $\bar{w}_{1}=\left(\tilde{x}\left(t_{0}+1-\varepsilon^{\delta}, z, \varepsilon\right)+a_{0}\right) / \varepsilon, \bar{w}_{2}=\tilde{y}\left(t_{0}+1-\varepsilon^{\delta}, z, \varepsilon\right)$, переменная $\tau$ по-прежнему меняется на отрезке $J(\varepsilon)$, а добавки $H_{1}, H_{2}$ определены равенствами (3.30), в которых, естественно, вместо функций (3.26) фигурируют их аналоги из (3.47).

Как и выше, наряду с (3.52) рассмотрим соответствующую предельную систему. Из соотношений (3.30), (3.49)-(3.51) вытекает, что в данном случае эта система записывается в виде

$$
\begin{aligned}
& \frac{d w_{1}}{d \tau}=f\left(\exp \left(-a_{0} \tau+c_{0}+\theta_{1}\right)\right), \\
& \frac{d w_{2}}{d \tau}=f\left(\exp \left(-a_{0} \tau+c_{0}+\theta_{2}\right)\right)-f\left(\exp \left(-a_{0} \tau+c_{0}+\theta_{1}\right)\right),
\end{aligned}
$$

где функции $\theta_{j}=\theta_{j}(z), j=1,2$, задаются формулами

$$
\begin{aligned}
\theta_{1}=d & \int_{-\sigma_{0}}^{t_{0}}\left[\exp \left(y_{0}(s, z)\right)-1\right] d s \\
& -\left(1+a_{0}\right) d \int_{-\sigma_{0}}^{0}\left[\exp \left(y_{0}(s, z)\right)-1\right] d s, \quad \theta_{2}=\theta_{1}+y_{0}\left(t_{0}, z\right) .
\end{aligned}
$$

Непосредственная проверка показывает, что система (3.53) имеет решение с компонентами

$$
w_{1}^{0}(\tau, z)=w\left(\tau-\frac{\theta_{1}}{a_{0}}\right)+\theta_{3}, \quad w_{2}^{0}(\tau, z)=w\left(\tau-\frac{\theta_{2}}{a_{0}}\right)-w\left(\tau-\frac{\theta_{1}}{a_{0}}\right)+\theta_{4},
$$

где

$$
\theta_{3}=d \int_{t_{0}}^{t_{0}+1}\left[\exp \left(y_{0}(s, z)\right)-1\right] d s, \quad \theta_{4}=y_{0}\left(t_{0}+1-0, z\right)-y_{0}\left(t_{0}, z\right),
$$

а $w(\tau)$ - функция (2.34). Как и в предыдущем случае, выбор именно этого решения продиктован двумя обстоятельствами: оно с точностью до $O\left(\varepsilon^{\delta}\right)$ 
удовлетворяет начальным условиям из (3.52) и обладает аналогичными (3.45) свойствами

$$
\begin{array}{rlrl}
w_{2}^{0} & =y_{0}\left(t_{0}+1-0, z\right)+O\left(\exp \left(a_{0} \tau\right)\right), & \tau \rightarrow-\infty \\
\frac{\partial w_{2}^{0}}{\partial z}=\frac{\partial y_{0}}{\partial z}\left(t_{0}+1-0, z\right)+O\left(\exp \left(a_{0} \tau\right)\right), & \tau \rightarrow-\infty \\
w_{2}^{0}=y_{0}\left(t_{0}+1+0, z\right)+O\left(\exp \left(-a_{0} \tau\right)\right), & \tau \rightarrow+\infty \\
\frac{\partial w_{2}^{0}}{\partial z}=\frac{\partial y_{0}}{\partial z}\left(t_{0}+1+0, z\right)+O\left(\exp \left(-a_{0} \tau\right)\right), & \tau \rightarrow+\infty
\end{array}
$$

Более того, справедливо следующее утверждение.

Лемма 3.2. Для решения $\left(w_{1}(\tau, z, \varepsilon), w_{2}(\tau, z, \varepsilon)\right)$ задачи Коши (3.52) равномерно по $\tau \in J(\varepsilon)$ и по параметру z выполняются асимптотические равенства

$$
w_{j}(\tau, z, \varepsilon)=w_{j}^{0}(\tau, z)+O\left(\varepsilon^{\delta}\right), \quad \frac{\partial w_{j}}{\partial z}(\tau, z, \varepsilon)=\frac{\partial w_{j}^{0}}{\partial z}(\tau, z)+O\left(\varepsilon^{\delta}\right), \quad j=1,2,
$$

где $w_{j}^{0}, j=1,2,-$ функиии, определяемые формулами (3.54)-(3.57).

На доказательстве этой леммы не останавливаемся, поскольку оно с несущественными изменениями повторяет обоснование леммы 3.1. Приведем сразу вытекающие из (3.58) итоговые формулы

$$
\begin{aligned}
& x_{\varphi}(t, \varepsilon)=-a_{0}+\left.\varepsilon w_{1}^{0}(\tau, z)\right|_{\tau=\left(t-t_{0}-1\right) / \varepsilon}+O\left(\varepsilon^{1+\delta}\right), \\
& y_{\varphi}(t, \varepsilon)=\left.w_{2}^{0}(\tau, z)\right|_{\tau=\left(t-t_{0}-1\right) / \varepsilon}+O\left(\varepsilon^{\delta}\right)
\end{aligned}
$$

равномерные по $t \in\left[t_{0}+1-\varepsilon^{\delta}, t_{0}+1+\varepsilon^{\delta}\right], \varphi \in S$.

Заключительный этап построения асимптотики решения $\left(x_{\varphi}(t, \varepsilon), y_{\varphi}(t, \varepsilon)\right)$ связан с рассмотрением отрезка $t_{0}+1+\varepsilon^{\delta} \leqslant t \leqslant T_{0}+\varepsilon^{\delta}$. Применяя уже неоднократно упоминавшийся метод шагов, последовательно убеждаемся, что при указанных $t$ имеют место оценка $x_{\varphi}(t-1, \varepsilon) \leqslant-M \varepsilon^{\delta}, M=$ const $>0$, и соответствующие формулы (с точностью до $O\left(\exp \left(-q / \varepsilon^{1-\delta}\right)\right), q=$ const $\left.>0\right)$

$$
x_{\varphi}(t, \varepsilon)=\tilde{x}(t, z, \varepsilon), \quad y_{\varphi}(t, \varepsilon)=\tilde{y}(t, z, \varepsilon),
$$

где $(\tilde{x}, \tilde{y})$ - решение задачи Коши

$$
\begin{gathered}
\dot{x}=\varepsilon d(\exp y-1)+1, \quad \dot{y}=-2 d \operatorname{sh} y \\
\left.x\right|_{t=t_{0}+1+\varepsilon^{\delta}}=-a_{0}+\left.\varepsilon w_{1}(\tau, z, \varepsilon)\right|_{\tau=\varepsilon^{\delta-1}},\left.\quad y\right|_{t=t_{0}+1+\varepsilon^{\delta}}=\left.w_{2}(\tau, z, \varepsilon)\right|_{\tau=\varepsilon^{\delta-1}} .
\end{gathered}
$$

Несложный анализ задачи (3.61), опирающийся на равенства (3.58), приводит к асимптотическим представлениям

$$
\begin{gathered}
\tilde{x}=t-T_{0}+\varepsilon d \int_{t_{0}+1}^{t}\left[\exp \left(y_{0}(s, z)\right)-1\right] d s+\varepsilon\left(\theta_{3}(z)-\frac{\theta_{1}(z)}{a_{0}}\right)+O\left(\varepsilon^{1+\delta}\right), \\
\tilde{y}=y_{0}(t, z)+O\left(\varepsilon^{\delta}\right)
\end{gathered}
$$


справедливым равномерно по $t, z$ и допускающим дифференцирование по переменной $z$.

Подведем промежуточный итог. Из формул (3.60), (3.62) и очевидного равенства $\dot{x}_{\varphi}(t, \varepsilon)=1+O(\varepsilon)$, равномерного по $t \in\left[t_{0}+1+\varepsilon^{\delta}, T_{0}+\varepsilon^{\delta}\right], \varphi \in S$, заключаем, что требуемый корень $t=T_{\varphi}$ уравнения $x_{\varphi}\left(t-\sigma_{0}, \varepsilon\right)=-\sigma_{0}$ находится однозначно и допускает равномерную по $\varphi \in S$ асимптотику

$$
T_{\varphi}=T_{0}+O(\varepsilon)
$$

где $T_{0}$ - величина (1.8). А отсюда, в свою очередь, вытекает, что оператор (3.9) заведомо определен на множестве $S$ при всех $\varepsilon \in\left(0, \varepsilon_{0}\right]$, где $\varepsilon_{0}=\varepsilon_{0}(S)>0$ достаточно мало.

На следующем этапе просуммируем всю установленную выше информацию об асимптотическом поведении решения $\left(x_{\varphi}(t, \varepsilon), y_{\varphi}(t, \varepsilon)\right)$ (см. (3.24), (3.26), (3.46), (3.47), (3.49)-(3.51), (3.59), (3.60), (3.62), (3.63)). В результате убеждаемся, что равномерно по $\varphi \in S$

$$
\begin{gathered}
\max _{-\sigma_{0} \leqslant t \leqslant T_{\varphi}-\sigma_{0}}\left|x_{\varphi}(t, \varepsilon)-x_{0}(t)\right|=O(\varepsilon), \\
\max _{t \in \Sigma(\varepsilon)}\left|y_{\varphi}(t, \varepsilon)-y_{0}(t, z)\right|_{z=\varphi_{2}\left(-\sigma_{0}\right)} \mid=O\left(\varepsilon^{\delta}\right),
\end{gathered}
$$

где $x_{0}(t)$ - функция (2.9), $y_{0}(t, z)$ - решение задачи $(3.11),(3.12)$, а множество $\Sigma(\varepsilon)$ представляет собой отрезок $\left[-\sigma_{0}, T_{\varphi}-\sigma_{0}\right]$ с выброшенными интервалами $\left(1-\varepsilon^{\delta}, 1+\varepsilon^{\delta}\right),\left(t_{0}+1-\varepsilon^{\delta}, t_{0}+1+\varepsilon^{\delta}\right)$. И наконец, объединяя формулы $(3.64),(3.65)$, приходим к выводу о справедливости первого предельного равенства из (3.21).

Доказательство второго предельного соотношения из (3.21) помимо уже установленных асимптотических свойств решения $\left(x_{\varphi}(t, \varepsilon), y_{\varphi}(t, \varepsilon)\right)$ требует знания асимптотики при $-\sigma_{0} \leqslant t \leqslant T_{\varphi}-\sigma_{0}$ решения $\left(g_{1}(t, \varepsilon), g_{2}(t, \varepsilon)\right)$ линейной системы (3.16) с произвольной начальной функцией $g_{0}(t)$ из пространства $\mathscr{F}_{0}$. Соответствующий анализ приводится ниже.

Как обычно, начнем с промежутка $-\sigma_{0} \leqslant t \leqslant 1-\sigma_{0}$. Учитывая в коэффициентах (3.17)-(3.19) явный вид функций $F, G$ (см. (2.1), (3.6)) и формулы (3.24), (3.26), приходим к выводу, что равномерно по $t \in\left[-\sigma_{0}, 1-\sigma_{0}\right], \varphi \in S$

$$
\begin{gathered}
a_{11}=O\left(\exp \left(-\frac{q}{\varepsilon}\right)\right), \quad a_{12}=\varepsilon d \exp (\tilde{y}(t, z, \varepsilon))+O\left(\exp \left(-\frac{q}{\varepsilon}\right)\right), \\
a_{21}=O\left(\exp \left(-\frac{q}{\varepsilon}\right)\right), \quad a_{22}=-2 d \operatorname{ch}(\tilde{y}(t, z, \varepsilon))+O\left(\exp \left(-\frac{q}{\varepsilon}\right)\right), \\
a_{23}=O\left(\exp \left(-\frac{q}{\varepsilon}\right)\right), \quad q=\text { const }>0 .
\end{gathered}
$$

А отсюда, в свою очередь, следует, что на рассматриваемом отрезке

$$
g_{1}=\frac{\partial \tilde{x}}{\partial z}(t, z, \varepsilon) g_{2,0}\left(-\sigma_{0}\right)+\tilde{g}_{1}(t, \varepsilon), \quad g_{2}=\frac{\partial \tilde{y}}{\partial z}(t, z, \varepsilon) g_{2,0}\left(-\sigma_{0}\right)+\tilde{g}_{2}(t, \varepsilon),
$$

где $(\tilde{x}, \tilde{y})$ - решение задачи Коши (3.25), а добавки $\tilde{g}_{1}, \tilde{g}_{2}$ удовлетворяют оценкам вида

$$
\max _{t}\left|\tilde{g}_{j}(t, \varepsilon)\right| \leqslant M \exp \left(-\frac{q}{\varepsilon}\right)\left\|g_{0}\right\|_{\mathscr{F}_{0}}, \quad j=1,2,
$$


с некоторыми универсальными (не зависящими от $\varepsilon, \varphi, g_{0}$ ) положительными постоянными $M$ и $q$.

Обратимся, далее, к отрезку $1-\sigma_{0} \leqslant t \leqslant 1-\varepsilon^{\delta}$. Снова опираясь на асимптотические представления (3.24), (3.26), замечаем, что на нем формулы (3.66) сохраняются, но с остатками порядка $\exp \left(-q / \varepsilon^{1-\delta}\right), q=$ const $>0$. А это означает, что при указанных $t$ сохраняются и асимптотические равенства (3.67). Однако теперь для соответствующих добавок $\tilde{g}_{j}(t, \varepsilon), j=1,2$, вместо (3.68) справедливы оценки вида

$$
\max _{t}\left|\tilde{g}_{j}(t, \varepsilon)\right| \leqslant M \exp \left(-\frac{q}{\varepsilon^{1-\delta}}\right)\left\|g_{0}\right\|_{\mathscr{F}_{0}}, \quad j=1,2 .
$$

На очередном отрезке $1-\varepsilon^{\delta} \leqslant t \leqslant 1+\varepsilon^{\delta}$ функции $g_{j}(t-1, \varepsilon), j=1,2$, задаются уже известными равенствами (3.67). Учитывая данное обстоятельство и опираясь на установленные ранее асимптотические представления для $x_{\varphi}(t-1, \varepsilon), y_{\varphi}(t-1, \varepsilon), y_{\varphi}(t, \varepsilon)$ (см. (3.24), (3.26), (3.46)), приходим к выводу, что при $t \in\left[1-\varepsilon^{\delta}, 1+\varepsilon^{\delta}\right]$ с точностью до величин порядка $\exp \left(-q / \varepsilon^{1-\delta}\right)$, $q=$ const $>0$, компоненты $g_{1}(t, \varepsilon), g_{2}(t, \varepsilon)$ определяются из задачи Коши

$$
\begin{gathered}
\dot{g}_{1}=\varepsilon d \exp \left\{\left.v_{2}(\tau, z, \varepsilon)\right|_{\tau=(t-1) / \varepsilon}\right\} g_{2}+g_{2,0}\left(-\sigma_{0}\right) \frac{\partial}{\partial z}\left(F_{z}(t, \varepsilon)\right), \\
\dot{g}_{2}=-2 d \operatorname{ch}\left\{\left.v_{2}(\tau, z, \varepsilon)\right|_{\tau=(t-1) / \varepsilon}\right\} g_{2}+g_{2,0}\left(-\sigma_{0}\right) \frac{\partial}{\partial z}\left(G_{z}(t, \varepsilon)\right), \\
\left.g_{1}\right|_{t=1-\varepsilon^{\delta}}=g_{2,0}\left(-\sigma_{0}\right) \frac{\partial}{\partial z}\left(x_{z}(\varepsilon)\right),\left.\quad g_{2}\right|_{t=1-\varepsilon^{\delta}}=g_{2,0}\left(-\sigma_{0}\right) \frac{\partial}{\partial z}\left(y_{z}(\varepsilon)\right),
\end{gathered}
$$

где $F_{z}, G_{z}, x_{z}(\varepsilon), y_{z}(\varepsilon)$ - функции $(3.28), v_{2}(\tau, z, \varepsilon)$ - компонента решения задачи Коши (3.29). А отсюда и из того факта, что (3.70) представляет собой “продифференцированную по $z$ ” задачу (3.27), окончательно получаем:

$$
\begin{aligned}
& g_{1}=\left.\varepsilon \frac{\partial v_{1}}{\partial z}(\tau, z, \varepsilon)\right|_{\tau=(t-1) / \varepsilon} \cdot g_{2,0}\left(-\sigma_{0}\right)+\tilde{g}_{1}(t, \varepsilon), \\
& g_{2}=\left.\frac{\partial v_{2}}{\partial z}(\tau, z, \varepsilon)\right|_{\tau=(t-1) / \varepsilon} \cdot g_{2,0}\left(-\sigma_{0}\right)+\tilde{g}_{2}(t, \varepsilon),
\end{aligned}
$$

где, как и ранее, остатки $\tilde{g}_{j}, j=1,2$, допускают оценки (3.69).

Дальнейший анализ приводит к аналогичным результатам. А именно, рассуждая как выше, последовательно убеждаемся, что, во-первых, при $t \in\left[1+\varepsilon^{\delta}\right.$, $\left.t_{0}+1-\varepsilon^{\delta}\right]$ сохраняют силу соотношения $(3.67),(3.69)$, в которых $(\tilde{x}, \tilde{y})-$ решение задачи Коши (3.48); во-вторых, на отрезке $t_{0}+1-\varepsilon^{\delta} \leqslant t \leqslant t_{0}+1+\varepsilon^{\delta}$ справедливы формулы, получающиеся из (3.71) при $\tau=\left(t-t_{0}-1\right) / \varepsilon$ и при замене функций $v_{1}, v_{2}$ компонентами $w_{1}, w_{2}$ решения задачи Коши (3.52); в-третьих, на оставшемся промежутке времени $t_{0}+1+\varepsilon^{\delta} \leqslant t \leqslant T_{0}+\varepsilon^{\delta}$ вновь имеем дело с равенствами (3.67), в которых теперь $(\tilde{x}, \tilde{y})$ - решение задачи Коши (3.61), а добавки $\tilde{g}_{j}, j=1,2$, по-прежнему удовлетворяют оценкам (3.69).

Полученная информация позволяет уже достаточно просто завершить обоснование теоремы о $C^{1}$-сходимости. Действительно, при $T_{\varphi}-\sigma_{0}-1 \leqslant t \leqslant T_{\varphi}-\sigma_{0}$ в силу неравенства $T_{\varphi}-\sigma_{0}-1>t_{0}+1+\varepsilon^{\delta}$ (вытекающего из (3.4), (3.64) 
и из условия $\left.\sigma_{0}<a_{0}-1\right)$ для $g_{1}(t, \varepsilon), g_{2}(t, \varepsilon)$ работают асимптотические представления (3.67), в которых, в свою очередь, функции $\tilde{x}, \tilde{y}$ задаются асимптотическими формулами (3.62), (3.63). А отсюда очевидным образом имеем

$$
\begin{gathered}
\max _{-1-\sigma_{0} \leqslant t \leqslant-\sigma_{0}}\left|g_{1}\left(t+T_{\varphi}, \varepsilon\right)\right| \leqslant M_{1} \varepsilon\left\|g_{0}\right\|_{\mathscr{F}_{0}}, \\
\max _{-1-\sigma_{0} \leqslant t \leqslant-\sigma_{0}}\left|g_{2}\left(t+T_{\varphi}, \varepsilon\right)-g_{2,0}\left(-\sigma_{0}\right) \frac{\partial y_{0}}{\partial z}\left(t+T_{0}, z\right)\right|_{z=\varphi_{2}\left(-\sigma_{0}\right)} \mid \leqslant M_{2} \varepsilon^{\delta}\left\|g_{0}\right\|_{\mathscr{F}_{0}},
\end{gathered}
$$

где постоянные $M_{1}, M_{2}>0$ не зависят от $\varepsilon, \varphi, g_{0}$. И наконец, применяя оценки (3.72) непосредственно к оператору $\partial_{\varphi} \Pi_{\varepsilon}(\varphi)$, задающемуся формулами (3.14), (3.20), убеждаемся в справедливости второго предельного равенства из (3.21). Теорема 3.1 полностью доказана.

Завершая обсуждение асимптотических свойств решения $\left(x_{\varphi}(t, \varepsilon), y_{\varphi}(t, \varepsilon)\right)$, приведем два следствия из теоремы 3.1. В связи с этим рассмотрим аналогичную (3.11), (3.12) импульсную систему

$$
\begin{gathered}
\dot{y}=-2 d \operatorname{sh} y, \\
y\left(t_{2 k-1}+0\right)=y\left(t_{2 k-1}-0\right)-\left(1+a_{0}\right) y\left(t_{2 k-1}-1\right), \quad k=1,2, \ldots, \\
y\left(t_{2 k}+0\right)=y\left(t_{2 k}-0\right)-\left(1+1 / a_{0}\right) y\left(t_{2 k}-1\right), \quad k=1,2, \ldots,
\end{gathered}
$$

где

$$
t_{2 k-1}=1+(k-1) T_{0}, \quad t_{2 k}=t_{0}+1+(k-1) T_{0}, \quad k \in \mathbb{N} .
$$

Решение данной системы на полуоси $t \in\left[-\sigma_{0},+\infty\right)$ с начальным условием $\left.y\right|_{t=-\sigma_{0}}=z, z \in \mathbb{R}$, как и ранее, обозначим через $y_{0}(t, z)$.

СЛЕДСТвИЕ 3.1.1. При любом фиксированном $T>0$ равномерно по $\varphi \in S$ выполняются предельные равенства

$$
\begin{gathered}
\lim _{\varepsilon \rightarrow 0} \max _{-\sigma_{0} \leqslant t \leqslant T-\sigma_{0}}\left|x_{\varphi}(t, \varepsilon)-x_{0}(t)\right|=0, \\
\lim _{\varepsilon \rightarrow 0} \max _{t \in \Sigma_{\varepsilon}(T)}\left|y_{\varphi}(t, \varepsilon)-y_{0}(t, z)\right|_{z=\varphi_{2}\left(-\sigma_{0}\right)} \mid=0 .
\end{gathered}
$$

Здесъ множество $\Sigma_{\varepsilon}(T)$ - отрезок $-\sigma_{0} \leqslant t \leqslant T-\sigma_{0}$ с выброшенными интервалами вида $\left(t_{2 k-1}-\varepsilon^{\delta}, t_{2 k-1}+\varepsilon^{\delta}\right),\left(t_{2 k}-\varepsilon^{\delta}, t_{2 k}+\varepsilon^{\delta}\right), \quad k=1,2, \ldots, \delta=$ const $\in(0,1)$.

Обращаем внимание, что при $T=T_{\varphi}$ справедливость равенств (3.74) уже установлена (см. (3.65)). В общем же случае разработанный выше алгоритм построения асимптотики решения $\left(x_{\varphi}(t, \varepsilon), y_{\varphi}(t, \varepsilon)\right)$ необходимо применить к отрезку $-\sigma_{0} \leqslant t \leqslant T-\sigma_{0}$.

Заслуживает внимания вопрос о справедливости аналога теоремы 3.1 при $a_{0}<1$. Для формулировки соответствующего результата зафиксируем постоянную $\sigma_{0}: 0<\sigma_{0}<a_{0}$ и обозначим через $\left(x_{z}(t, \varepsilon), y_{z}(t, \varepsilon)\right), t \geqslant-\sigma_{0}$, решение системы $(3.5)$ с начальными функциями $x_{z}(t, \varepsilon) \equiv-\sigma_{0}, y_{z}(t, \varepsilon) \equiv z$ при $-1-\sigma_{0} \leqslant t \leqslant-\sigma_{0}$, где $z=$ const $\in \mathbb{R}$. Далее, введем в рассмотрение второй положительный корень $t=T_{z}$ уравнения $x_{z}\left(t-\sigma_{0}, \varepsilon\right)=-\sigma_{0}$ и оператор

$$
\widetilde{\Pi}_{\varepsilon}(\varphi)=\left.\left(x_{z}\left(t+T_{z}, \varepsilon\right), y_{z}\left(t+T_{z}, \varepsilon\right)\right)\right|_{z=\varphi_{2}\left(-\sigma_{0}\right)}, \quad-1-\sigma_{0} \leqslant t \leqslant-\sigma_{0},
$$


действующий из множества (3.8) в пространство $\mathscr{F}$ и являющийся в некотором смысле аналогом предельного оператора (3.10). Предпринятый выше асимптотический анализ приводит к следующему утверждению.

СлЕДСТВИЕ 3.1.2. Имеют место аналогичные (3.21) пределъные равенства

$$
\begin{gathered}
\lim _{\varepsilon \rightarrow 0} \sup _{\varphi \in S}\left\|\Pi_{\varepsilon}(\varphi)-\widetilde{\Pi}_{\varepsilon}(\varphi)\right\|_{\mathscr{F}}=0, \\
\lim _{\varepsilon \rightarrow 0} \sup _{\varphi \in S}\left\|\partial_{\varphi} \Pi_{\varepsilon}(\varphi)-\partial_{\varphi} \widetilde{\Pi}_{\varepsilon}(\varphi)\right\|_{\mathscr{F}_{0} \rightarrow \mathscr{F}_{0}}=0 .
\end{gathered}
$$

Подчеркнем, что приведенные равенства отражают так называемый эффект забывания начальных условий: асимптотика решений системы (3.5) с точностью до величин порядка $\exp (-q / \varepsilon), q=$ const $>0$, не зависит от конкретного вида начального условия $\varphi \in S$, а определяется лишь значением функционала $z=\varphi_{2}\left(-\sigma_{0}\right)$. Тем самым, эти равенства справедливы при любом $a_{0}>0$. В случае же $a_{0}>1$ оператор $\widetilde{\Pi}_{\varepsilon}(\varphi)$, в свою очередь, сходится при $\varepsilon \rightarrow 0 \mathrm{~K} \Pi_{0}(\varphi)$ (в $C^{1}$-метрике) и мы можем перейти к более простым соотношениям (3.21).

При $a_{0}<1$, хотя упомянутый выше переход и невозможен, тем не менее, аналог теоремы 3.2 о соответствии остается в силе. Причина этого в том, что "предельный" оператор $\widetilde{\Pi}_{\varepsilon}(\varphi)$ является надстройкой (точный смысл этому термину придан в п. 3.1) над соответствующим одномерным отображением $\left.z \rightarrow y_{z}(t, \varepsilon)\right|_{t=T_{z}-\sigma_{0}}$, где $z=\varphi_{2}\left(-\sigma_{0}\right)$. Последнее же при $\varepsilon \rightarrow 0$ стремится (в $C^{1}$-метрике на любом конечном отрезке изменения $z$ ) к введенному выше отображению (3.22).

3.3. Анализ предельного отображения. В данном пункте обратимся к актуальному в связи с теоремой 3.2 вопросу о количестве и устойчивости неподвижных точек отображения (3.22).

Заметим в первую очередь, что интересующее нас отображение представляет собой оператор сдвига по траекториям системы (3.73) с $T_{0}$-периодическим импульсным воздействием за отрезок времени $-\sigma_{0} \leqslant t \leqslant T_{0}-\sigma_{0}$. А это означает, что его можно записать в инвариантной форме, заменяя указанный выше отрезок не зависящим от $\sigma_{0}$ промежутком $0 \leqslant t \leqslant T_{0}$.

Действительно, введем в рассмотрение оператор сдвига

$$
P^{t}(z)=\ln \frac{1+\operatorname{th}(z / 2) \exp (-2 d t)}{1-\operatorname{th}(z / 2) \exp (-2 d t)}, \quad t \in \mathbb{R}, \quad P^{0}(z)=z
$$

по решениям скалярного уравнения $\dot{y}=-2 d \operatorname{sh} y$ и положим $\bar{z}=\Phi(z)$. Тогда отображение (3.22) может быть представлено в виде

$$
\bar{z}=\left(P^{T_{0}-t_{0}-1-\sigma_{0}} \circ P_{2} \circ P^{t_{0}} \circ P_{1} \circ P^{1+\sigma_{0}}\right)(z),
$$

где через $P_{1}, P_{2}$ обозначены операторы пересчета начальных условий в точках $t=1$ и $t=t_{0}+1$ соответственно, действующие по правилам

$$
P_{1}(z)=z-\left(1+a_{0}\right) P^{-1}(z), \quad P_{2}(z)=z-\left(1+\frac{1}{a_{0}}\right) P^{-1}(z) .
$$


Далее, применим к левой и правой части получившегося равенства оператор $P^{\sigma_{0}}$. В результате с учетом очевидных соотношений

$$
P^{1+\sigma_{0}}=P^{1} \circ P^{\sigma_{0}}, \quad P^{T_{0}-t_{0}-1-\sigma_{0}}=P^{-\sigma_{0}} \circ P^{T_{0}-t_{0}-1}
$$

имеем

$$
P^{\sigma_{0}}(\bar{z})=\left(P^{T_{0}-t_{0}-1} \circ P_{2} \circ P^{t_{0}} \circ P_{1} \circ P^{1}\right)\left(P^{\sigma_{0}}(z)\right) .
$$

А отсюда, в свою очередь, следует, что после замены $P^{\sigma_{0}}(z) \rightarrow z$ интересующее нас отображение принимает требуемую инвариантную форму

$$
z \rightarrow \Phi_{0}(z) \stackrel{\text { def }}{=}\left(P^{T_{0}-t_{0}-1} \circ P_{2} \circ P^{t_{0}} \circ P_{1} \circ P^{1}\right)(z)
$$

или, что то же самое,

$$
\left.z \rightarrow \Phi_{0}(z) \stackrel{\text { def }}{=} y_{0}(t, z)\right|_{t=T_{0}},
$$

где $y_{0}(t, z), 0 \leqslant t \leqslant T_{0},-$ решение аналогичной (3.11), (3.12) задачи Коши

$$
\begin{gathered}
\dot{y}=-2 d \operatorname{sh} y,\left.\quad y\right|_{t=0}=z, \\
y(1+0)=y(1-0)-\left(1+a_{0}\right) y(0), \\
y\left(t_{0}+1+0\right)=y\left(t_{0}+1-0\right)-\left(1+\frac{1}{a_{0}}\right) y\left(t_{0}\right) .
\end{gathered}
$$

Остановимся на некоторых свойствах отображения (3.78). Привлекая его явный вид (3.77) и учитывая формулы (3.75), (3.76), нетрудно убедиться, что

$$
\begin{gathered}
\Phi_{0}(-z)=-\Phi_{0}(z), \quad \Phi_{0}(z)>0 \quad \forall z \in(0,+\infty) \\
\lim _{z \rightarrow+\infty} \Phi_{0}(z)=\Phi_{\infty}>0, \quad \Phi_{\infty}=\ln \frac{1+\operatorname{th}\left(\gamma_{\infty} / 2\right) \exp \left(-2 d a_{0}\right)}{1-\operatorname{th}\left(\gamma_{\infty} / 2\right) \exp \left(-2 d a_{0}\right)} \\
\gamma_{\infty}=\ln \left[\operatorname{th}\left(d\left(1+\frac{1}{a_{0}}\right)\right)\right]-\left(1+\frac{1}{a_{0}}\right) \ln \left[\operatorname{th} \frac{d}{a_{0}}\right]
\end{gathered}
$$

Заметим, далее, что нечетность функции $\Phi_{0}(z)$ (см. (3.80)) обусловлена инвариантностью исходной системы (3.2) по отношению к преобразованию координат (3.3). Что же касается свойства (3.81), то оно гарантирует диссипативность отображения (3.78) на инвариантной полуоси $z \in \mathbb{R}_{+}$.

Перейдем теперь непосредственно к анализу возможных аттракторов рассматриваемого отображения. Непосредственная проверка показывает, что оно имеет нулевую неподвижную точку, причем

$$
\Phi_{0}^{\prime}(0)=\left.h(t)\right|_{t=T_{0}}
$$

где $h(t)$ - решение аналогичной (3.79) линеаризованной задачи

$$
\begin{gathered}
\dot{h}=-2 d h,\left.\quad h\right|_{t=0}=1, \\
h(1+0)=h(1-0)-\left(1+a_{0}\right) h(0), \\
h\left(t_{0}+1+0\right)=h\left(t_{0}+1-0\right)-\left(1+\frac{1}{a_{0}}\right) h\left(t_{0}\right) .
\end{gathered}
$$


Далее, из (3.82), (3.83) заключаем, что

$$
\begin{gathered}
\Phi_{0}^{\prime}(0)=\left.\mu(s)\right|_{s=2 d}, \\
\mu(s)=\left(\left(1+a_{0}\right) \exp s-1\right)\left(\left(1+\frac{1}{a_{0}}\right) \exp s-1\right) \exp \left(-s T_{0}\right) .
\end{gathered}
$$

И наконец, опираясь на очевидные свойства функции $\mu(s)$ :

$$
\begin{gathered}
\mu(0)=1, \\
\mu^{\prime}(s)=\left(\left(2-T_{0}\right) \exp (2 s)+\left(T_{0}-1\right) \exp s-1\right) T_{0} \exp \left(-s T_{0}\right)<0 \quad \forall s \in(0,+\infty), \\
\mu(s) \rightarrow 0 \quad \text { при } s \rightarrow+\infty,
\end{gathered}
$$

приходим к выводу, что $0<\mu(s)<1$ при любом $s \in(0,+\infty)$. Таким образом, нулевая неподвижная точка отображения (3.78) оказывается экспоненциально устойчивой при любом $d>0$.

В рамках исходной билокальной модели (3.1) неподвижной точке $z=0$ отвечает однородный цикл

$$
N_{1}=N_{2}=N_{*}(t, \lambda)
$$

периода $T_{*}(\lambda)$, где $N_{*}(t, \lambda), T_{*}(\lambda)$ определены равенствами $(2.58),(2.59)$. Остается воспользоваться теоремой 3.2 , из которой вытекает следующее утверждение.

Теорема 3.3. Однородный цикл (3.86) системы (3.1) является экспоненциально орбитально устойчивым при любом фиксированном значении коэффициента дифбузии $d>0$ и при всех $\lambda \geqslant \lambda_{0}$, где $\lambda_{0}=\lambda_{0}(d)>0$ достаточно велико.

Характерная особенность отображения (3.78) заключается в том, что при уменьшении параметра $d$ на его инвариантном множестве $\mathbb{R}_{+}$происходит бифуркация рождения пары неподвижных точек - устойчивой и неустойчивой. Наглядное представление об этом процессе дают графики функции $\Phi_{0}(z)$ при $a_{0}=5, d=0.09,0.07,0.05$, построенные с помощью формул (3.75)-(3.77) на отрезке $0 \leqslant z \leqslant 2$ (см. рис. 3.1-3.3). Заметим, далее, что в силу свойства нечетности $\Phi_{0}(z)$ устойчивой ненулевой неподвижной точке из $\mathbb{R}_{+}$отвечает аналогичная симметрично расположенная неподвижная точка из $\mathbb{R}_{-}$, а им обеим в исходной релаксационной системе (3.1) при всех достаточно больших $\lambda$ соответствует пара устойчивых циклов, переходящих друг в друга под действием замены $\left(N_{1}, N_{2}\right) \rightarrow\left(N_{2}, N_{1}\right)$. Добавим еще, что согласно теореме 3.3 эти циклы сосуществуют с устойчивым однородным циклом (3.86).

Некоторое представление о релаксационных свойствах упомянутых чуть выше устойчивых неоднородных циклов дает рис. 3.4, где представлены графики компонент $N_{1}(t), N_{2}(t)$ одного из них в случае нелинейности (1.7) при $c=0.5$ и при значениях параметров $\lambda=7.8, d=0.05$ (сплошной линией изображена функция $N_{1}(t)$, а пунктирной $\left.-N_{2}(t)\right)$.

Как оказывается, при малых $d$ устойчивая ненулевая неподвижная точка отображения (3.78), принадлежащая множеству $\mathbb{R}_{+}$, может быть найдена 


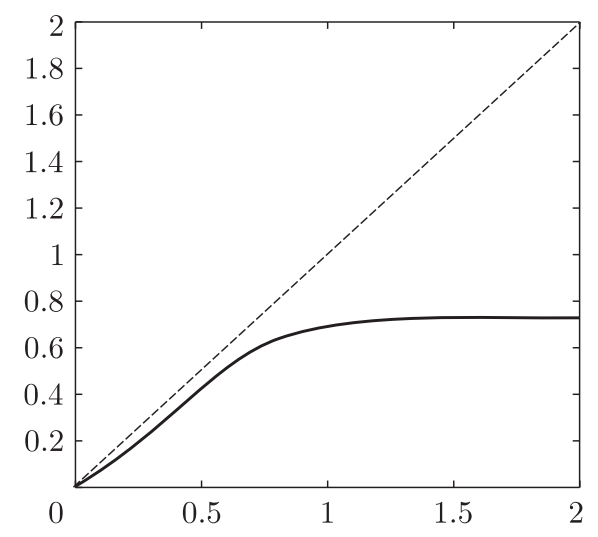

Рис. 3.1. $d=0.09$

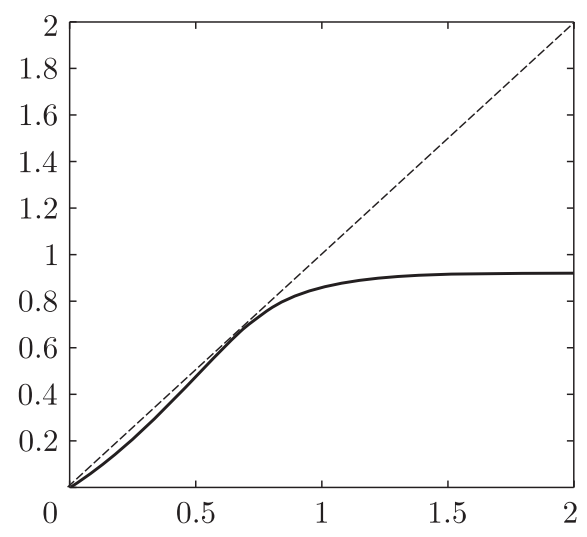

Рис. 3.2. $d=0.07$

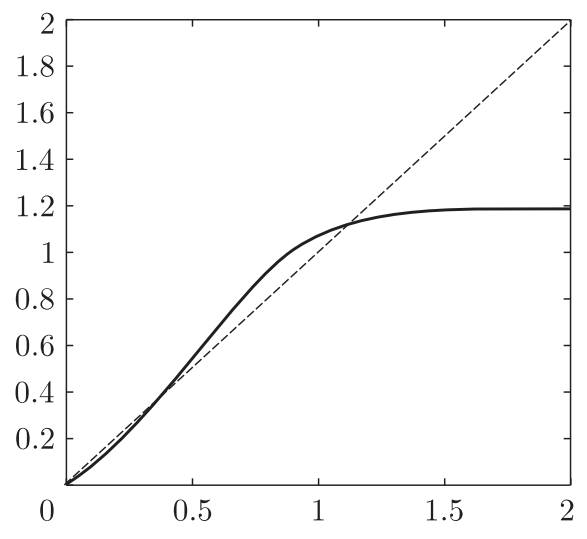

Рис. 3.3. $d=0.05$

асимптотическими методами. Действительно, считая по-прежнему выполненным неравенство (3.4), положим в системе (3.79)

$$
z=\frac{1}{a_{0}} \ln \frac{1}{d}+u, \quad u=\text { const } \in \mathbb{R}, \quad 0<d \ll 1 .
$$

В результате на полуинтервале $0 \leqslant t<1$ получаем задачу Коши

$$
\dot{y}=-2 d \operatorname{sh} y,\left.\quad y\right|_{t=0}=\frac{1}{a_{0}} \ln \frac{1}{d}+u,
$$

а ее решение будем искать в виде

$$
y(t, u, d)=\frac{1}{a_{0}} \ln \frac{1}{d}+h(t, u, d), \quad h(0, u, d)=u .
$$

Подставляя затем соотношение (3.89) в (3.88), приходим к выводу, что равномерно по $t \in[0,1)$

$$
h(t, u, d)=u+O\left(d^{1-1 / a_{0}}\right), \quad \frac{\partial h}{\partial u}(t, u, d)=1+O\left(d^{1-1 / a_{0}}\right) .
$$




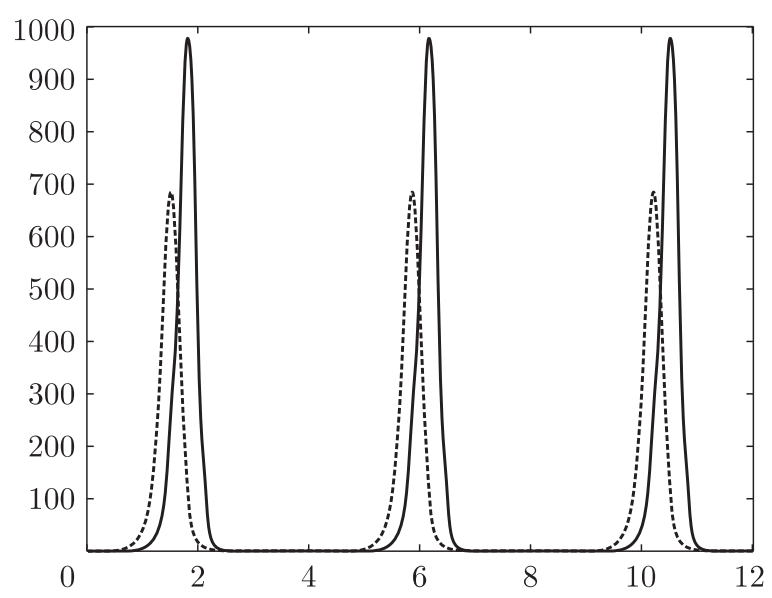

Рис. 3.4

Далее, в момент времени $t=1$ первый раз происходит скачок и согласно $(3.79),(3.89),(3.90)$ при $t \in\left[1, t_{0}+1\right)$ имеем дело с задачей Коши

$$
\dot{y}=-2 d \operatorname{sh} y,\left.\quad y\right|_{t=1}=-\ln \frac{1}{d}-a_{0} u+O\left(d^{1-1 / a_{0}}\right) .
$$

Как и выше, ее решение ищем в аналогичном (3.89) виде

$$
y(t, u, d)=-\ln \frac{1}{d}+h(t, u, d) .
$$

В итоге, с точностью до величин порядка $d^{1-1 / a_{0}}$, для функции $h(t, u, d)$ из (3.92) приходим к задаче Коши

$$
\dot{h}=\exp (-h),\left.\quad h\right|_{t=1}=-a_{0} u .
$$

А отсюда, в свою очередь, следует, что решение $y(t, u, d)$ исходной задачи $(3.91)$ допускает равномерные по $t \in\left[1, t_{0}+1\right)$ асимптотические представления

$$
\begin{aligned}
y(t, u, d) & =-\ln \frac{1}{d}+\ln \left(t-1+\exp \left(-a_{0} u\right)\right)+O\left(d^{1-1 / a_{0}}\right), \\
\frac{\partial y}{\partial u}(t, u, d) & =-\frac{a_{0} \exp \left(-a_{0} u\right)}{t-1+\exp \left(-a_{0} u\right)}+O\left(d^{1-1 / a_{0}}\right) .
\end{aligned}
$$

При $t=t_{0}+1$ происходит очередной скачок, причем в силу (3.93), (3.79) на оставшемся отрезке $t_{0}+1 \leqslant t \leqslant T_{0}$ получается задача Коши

$$
\dot{y}=-2 d \operatorname{sh} y,\left.\quad y\right|_{t=t_{0}+1}=\frac{1}{a_{0}} \ln \frac{1}{d}+\psi(u)+O\left(d^{1-1 / a_{0}}\right),
$$

где

$$
\psi(u)=\ln \left(1+\frac{1}{a_{0}}+\exp \left(-a_{0} u\right)\right)-\left(1+\frac{1}{a_{0}}\right) \ln \left(\frac{1}{a_{0}}+\exp \left(-a_{0} u\right)\right) .
$$


Опуская несложный ее анализ, приведем сразу вытекающие из (3.94), (3.95) окончательные асимптотические формулы

$$
y(t, u, d)=\frac{1}{a_{0}} \ln \frac{1}{d}+\psi(u)+O\left(d^{1-1 / a_{0}}\right), \quad \frac{\partial y}{\partial u}(t, u, d)=\psi^{\prime}(u)+O\left(d^{1-1 / a_{0}}\right),
$$

равномерные по $t \in\left[t_{0}+1, T_{0}\right]$.

Проделанные построения показывают, что после перехода к новой переменной $u$ (см. (3.87)) отображение (3.78) принимает вид

$$
u \rightarrow \psi(u)+\Delta(u, d)
$$

где

$$
\Delta(u, d)=\left.y(t, u, d)\right|_{t=T_{0}}-\frac{1}{a_{0}} \ln \frac{1}{d}-\psi(u) .
$$

Заметим, далее, что поскольку асимптотические представления (3.89), (3.90), (3.93), (3.96) выполняются равномерно по $u$ из любого конечного отрезка $\left[u_{1}, u_{2}\right]$, то и остаток (3.98) имеет при $d \rightarrow 0$ порядок малости $O\left(d^{1-1 / a_{0}}\right)$ в метрике пространства $C^{1}\left[u_{1}, u_{2}\right]$. Последнее же обстоятельство позволяет при рассмотрении интересующего нас вопроса о неподвижных точках отбросить упомянутый остаток и заменить (3.97) соответствующим предельным отображением

$$
u \rightarrow \psi(u)
$$

Для выявления свойств отображения (3.99) рассмотрим сначала несколько более общее отображение

$$
u \rightarrow \psi_{r_{1}, r_{2}}(u) \stackrel{\text { def }}{=} \ln \left(r_{1}+\exp \left(-a_{0} u\right)\right)-\left(1+\frac{1}{a_{0}}\right) \ln \left(r_{2}+\exp \left(-a_{0} u\right)\right)
$$

с двумя параметрами $r_{1}, r_{2}>0$, удовлетворяющими условию

$$
r_{1}>\left(1+\frac{1}{a_{0}}\right) r_{2}
$$

Справедливо следующее утверждение.

ЛЕмма 3.3. При выполнении неравенства (3.101) отображение (3.100) имеет единственную глобально экспоненииально устойчивую неподвижную mочку $u_{*}=u_{*}\left(r_{1}, r_{2}\right)$.

ДокАЗАТЕЛЬСтво. Отметим сначала вытекающие из явного вида функции $\psi_{r_{1}, r_{2}}(u)($ см. (3.100)) асимптотические свойства

$$
\begin{gathered}
\lim _{u \rightarrow+\infty} \psi_{r_{1}, r_{2}}(u)=\ln r_{1}-\left(1+\frac{1}{a_{0}}\right) \ln r_{2}, \\
\psi_{r_{1}, r_{2}}(u)=u+\left(r_{1}-\left(1+\frac{1}{a_{0}}\right) r_{2}\right) \exp \left(a_{0} u\right)+O\left(\exp \left(2 a_{0} u\right)\right), \quad u \rightarrow-\infty
\end{gathered}
$$

Учитывая, далее, в (3.102) условие (3.101), приходим к выводу, что при всех достаточно больших $u>0$ выполняется неравенство $\psi_{r_{1}, r_{2}}(u)<u$, а при всех 
достаточно больших по модулю отрицательных $u$ - неравенство $\psi_{r_{1}, r_{2}}(u)>u$. Таким образом, отображение (3.100) заведомо имеет хотя бы одну неподвижную точку $u=u_{*}$, являющуюся, очевидно, корнем уравнения

$$
\exp \left(u_{*}\right)=\frac{r_{1}+\exp \left(-a_{0} u_{*}\right)}{\left(r_{2}+\exp \left(-a_{0} u_{*}\right)\right)^{1+1 / a_{0}}} .
$$

Перейдем теперь к вопросу об устойчивости найденной неподвижной точки. В связи с этим обратим внимание на то, что требование (3.101) влечет выполнение неравенства $r_{1}>r_{2}$. Используя данный факт, убеждаемся, что, во-первых,

$$
\begin{aligned}
& \frac{d}{d u} \psi_{r_{1}, r_{2}}(u) \\
& \quad=\exp \left(-a_{0} u\right)\left(\frac{a_{0}\left(r_{1}-r_{2}\right)}{\left(r_{1}+\exp \left(-a_{0} u\right)\right)\left(r_{2}+\exp \left(-a_{0} u\right)\right)}+\frac{1}{r_{2}+\exp \left(-a_{0} u\right)}\right)>0
\end{aligned}
$$

для любого $u \in \mathbb{R}$; во-вторых, за устойчивость неподвижной точки $u=u_{*}$ отвечает мультипликатор

$$
\left.\mu_{*} \stackrel{\text { def }}{=} \frac{d}{d u} \psi_{r_{1}, r_{2}}(u)\right|_{u=u_{*}}=\left.\left(\frac{v_{*}+r_{2}}{v_{*}+r_{1}}\right)^{a_{0}}\left(1+\frac{a_{0}\left(r_{1}-r_{2}\right)}{v_{*}+r_{1}}\right)\right|_{v_{*}=\exp \left(-a_{0} u_{*}\right)},
$$

являющийся положительным (при выводе равенства (3.105) привлекались соотношения (3.103), (3.104)). Кроме того, учитывая, что $a_{0}>1$, и опираясь на очевидные оценки

$$
\left(\frac{v_{*}+r_{1}}{v_{*}+r_{2}}\right)^{a_{0}}=\left(1+\frac{r_{1}-r_{2}}{v_{*}+r_{2}}\right)^{a_{0}}>1+\frac{a_{0}\left(r_{1}-r_{2}\right)}{v_{*}+r_{2}}>1+\frac{a_{0}\left(r_{1}-r_{2}\right)}{v_{*}+r_{1}},
$$

нетрудно увидеть, что $\mu_{*}<1$.

Итак, мы убедились, что любая возможная неподвижная точка $u=u_{*}$ отображения (3.100) является экспоненциально устойчивой. А это значит, что на самом деле такая точка единственна и в силу монотонности нашего отображения (см. (3.104)) глобально устойчива. Лемма 3.3 доказана.

Возвращаясь к отображению (3.99), заметим, что оно является частным случаем отображения (3.100) при $r_{1}=1+1 / a_{0}, r_{2}=1 / a_{0}$, причем условие $(3.101)$ здесь эквивалентно требованию (3.4). Тем самым, в силу леммы 3.3 рассматриваемое отображение имеет единственную устойчивую неподвижную точку $u=u_{*}$. Далее, переходя от (3.99) к (3.97) и учитывая $C^{1}$-малость остатка (3.98), убеждаемся, что отображение (3.97) также допускает устойчивую неподвижную точку $u=u(d), u(0)=u_{*}$. И наконец, выражая $u$ через $z$ (см. (3.87)), приходим к выводу, что искомая ненулевая устойчивая неподвижная точка исходного отображения (3.78), принадлежащая множеству $\mathbb{R}_{+}$, задается равенством

$$
z(d)=\frac{1}{a_{0}} \ln \frac{1}{d}+u(d), \quad 0<d \ll 1 .
$$


Завершая исследование отображения (3.78), напомним, что помимо (3.106) у него имеется еще устойчивая неподвижная точка $z=-z(d)$. Напомним также, что согласно теореме 3.2 данной паре неподвижных точек в билокальной модели (3.2) отвечает пара устойчивых циклов, переходящих друг в друга в результате перестановки координат (3.3).

\section{4. Многомерный случай}

4.1. Теорема о $C^{1}$-сходимости. Развитые в предыдущем разделе асимптотические конструкции практически без изменений переносятся на диффузионную цепочку (1.10) при любом $m \geqslant 2$. Связано это с тем, что после перехода в $(1.10)$ к переменным $x, y_{1}, \ldots, y_{m-1}$, где

$$
N_{1}=\exp \left(\frac{x}{\varepsilon}\right), \quad N_{j}=\exp \left(\frac{x}{\varepsilon}+\sum_{k=1}^{j-1} y_{k}\right), \quad j=2, \ldots, m, \quad \varepsilon=\frac{1}{\lambda},
$$

получается аналогичная (3.5) релаксационная система

$$
\begin{aligned}
\dot{x}=\varepsilon d & \left(\exp y_{1}-1\right)+F(x(t-1), \varepsilon), \\
\dot{y}_{j}=d[ & \left.\exp y_{j+1}+\exp \left(-y_{j}\right)-\exp y_{j}-\exp \left(-y_{j-1}\right)\right] \\
& \quad+G_{j}\left(x(t-1), y_{1}(t-1), \ldots, y_{j}(t-1), \varepsilon\right), \quad j=1, \ldots, m-1 .
\end{aligned}
$$

Здесь $y_{0}=y_{m}=0$, функция $F(x, \varepsilon)$ та же самая, что и в $(2.1)$, а нелинейности $G_{j}$ задаются аналогичными (3.6) соотношениями

$$
\begin{aligned}
G_{j}\left(x, y_{1}, \ldots, y_{j}, \varepsilon\right)=\frac{1}{\varepsilon}\left\{f\left(\exp \left(\frac{x}{\varepsilon}+\sum_{k=1}^{j} y_{k}\right)\right)-f\left(\exp \left(\frac{x}{\varepsilon}+\sum_{k=1}^{j-1} y_{k}\right)\right)\right\} \\
j=1, \ldots, m-1 .
\end{aligned}
$$

Для того чтобы сформулировать в данном случае соответствующий вариант теоремы о $C^{1}$-сходимости, снова обратимся к наиболее простой ситуации, когда выполняется неравенство (3.4). Зафиксируем затем постоянную $\sigma_{0}$ такую, что $0<\sigma_{0}<a_{0}-1$, и обозначим через $\left(x_{\varphi}(t, \varepsilon), y_{1, \varphi}(t, \varepsilon), \ldots, y_{m-1, \varphi}(t, \varepsilon)\right)$, $t \geqslant-\sigma_{0}$, решение системы (4.1) с произвольным начальным условием $\varphi(t)=$ $\left(\varphi_{1}(t), \ldots, \varphi_{m}(t)\right),-1-\sigma_{0} \leqslant t \leqslant-\sigma_{0}$, из множества

$$
S=\left\{\left(\varphi_{1}(t), \ldots, \varphi_{m}(t)\right): \varphi_{1} \in S_{1}, \varphi_{2} \in S_{2}, \ldots, \varphi_{m} \in S_{m}\right\} \subset \mathscr{F} .
$$

Здесь $S_{1}$ - то же самое семейство функций, что и в $(3.8), S_{2}, \ldots, S_{m}$ - произвольные замкнутые и ограниченные подмножества из пространства $C\left[-1-\sigma_{0}\right.$, $\left.-\sigma_{0}\right]$, а $\mathscr{F}$ - банахово пространство непрерывных при $-1-\sigma_{0} \leqslant t \leqslant-\sigma_{0}$ вектор-функций $\varphi(t)=\left(\varphi_{1}(t), \ldots, \varphi_{m}(t)\right)$ с нормой $(3.7)$.

Далее, рассмотрим аналогичные (3.9) и (3.10) операторы

$$
\begin{aligned}
& \Pi_{\varepsilon}(\varphi)=\left(x_{\varphi}\left(t+T_{\varphi}, \varepsilon\right), y_{1, \varphi}\left(t+T_{\varphi}, \varepsilon\right), \ldots, y_{m-1, \varphi}(\right.\left.\left.t+T_{\varphi}, \varepsilon\right)\right), \\
&-1-\sigma_{0} \leqslant t \leqslant-\sigma_{0} ; \\
& \Pi_{0}(\varphi)=\left.\left(x_{0}(t), y_{1}^{0}\left(t+T_{0}, z\right), \ldots, y_{m-1}^{0}\left(t+T_{0}, z\right)\right)\right|_{z=\left(\varphi_{2}\left(-\sigma_{0}\right), \ldots, \varphi_{m}\left(-\sigma_{0}\right)\right)}, \\
&-1-\sigma_{0} \leqslant t \leqslant-\sigma_{0},
\end{aligned}
$$


где, как и в п. 3.1, через $t=T_{\varphi}$ обозначен второй положительный корень уравнения $x_{\varphi}\left(t-\sigma_{0}, \varepsilon\right)=-\sigma_{0}, T_{0}$ - величина, определенная в $(1.8)$, а $x_{0}(t)$ - периодическая функция (2.9). Что же касается функций $y_{1}^{0}(t, z), \ldots, y_{m-1}^{0}(t, z)$, где $z=\left(z_{1}, \ldots, z_{m-1}\right) \in \mathbb{R}^{m-1}, t \in\left[-\sigma_{0}, T_{0}-\sigma_{0}\right]$, то они удовлетворяют импульсной системе

$$
\begin{gathered}
\dot{y}_{j}=d\left[\exp y_{j+1}+\exp \left(-y_{j}\right)-\exp y_{j}-\exp \left(-y_{j-1}\right)\right], \\
y_{j}(1+0)=y_{j}(1-0)-\left(1+a_{0}\right) y_{j}(0), \quad j=1, \ldots, m-1, \quad y_{0}=y_{m}=0, \\
y_{j}\left(t_{0}+1+0\right)=y_{j}\left(t_{0}+1-0\right)-\left(1+\frac{1}{a_{0}}\right) y_{j}\left(t_{0}\right),
\end{gathered}
$$

дополненной начальным условием

$$
\left.\left(y_{1}, \ldots, y_{m-1}\right)\right|_{t=-\sigma_{0}}=\left(z_{1}, \ldots, z_{m-1}\right) .
$$

Аналогом теоремы 3.1 о $C^{1}$-сходимости для системы (4.1) является следующее утверждение.

Теорема 4.1. Пусть выполнено условие (3.4). Тогда найдется такое достаточно малое $\varepsilon_{0}>0$ (зависящее от выбора множества (4.2)), что при каждом $\varepsilon \in\left(0, \varepsilon_{0}\right]$ оператор (4.3) определен на $S$ и допускает предельные равенства вида (3.21), во втором из которых подпространство $\mathscr{F}_{0} \subset \mathscr{F}$ задано аналогичным (3.13) соотношением

$$
\mathscr{F}_{0}=\left\{g_{0}(t)=\left(g_{1,0}(t), \ldots, g_{m, 0}(t)\right) \in \mathscr{F}: g_{1,0}\left(-\sigma_{0}\right)=0\right\} .
$$

Подробное обоснование данной теоремы опустим, поскольку, как уже было сказано, все построения из п. 3.2 переносятся на систему (4.1) с несущественными модификациями, обусловленными размерностью вектора $\left(y_{1}, \ldots, y_{m-1}\right)$. Единственный новый момент, появляющийся в случае $m>2$ и нуждающийся в дополнительном анализе, связан с корректностью определения оператора (4.4) на множестве $S$.

Действительно, на промежутках $-\sigma_{0} \leqslant t<1,1 \leqslant t<t_{0}+1$ и $t_{0}+1 \leqslant t \leqslant$ $T_{0}-\sigma_{0}$ решение задачи Коши (4.5), (4.6) удовлетворяет нелинейной системе

$$
\begin{gathered}
\dot{y}_{j}=d\left[\exp y_{j+1}+\exp \left(-y_{j}\right)-\exp y_{j}-\exp \left(-y_{j-1}\right)\right], \\
j=1, \ldots, m-1, \quad y_{0}=y_{m}=0 .
\end{gathered}
$$

Таким образом, возникает вопрос о продолжимости решений последней на указанные промежутки времени, длины которых отнюдь не малы.

При $m=2$ поставленный вопрос тривиален, так как в этом случае система (4.7) переходит в скалярное уравнение $\dot{y}=-2 d \operatorname{sh} y$. В случае же $m>2$ ответ на него дается в следующем утверждении.

Лемма 4.1. Решение $\left(y_{1}(t), \ldots, y_{m-1}(t)\right)$ системы (4.7) с произвольным начальным условием $\left.\left(y_{1}, \ldots, y_{m-1}\right)\right|_{t=0}=\left(\bar{y}_{1}, \ldots, \bar{y}_{m-1}\right) \in \mathbb{R}^{m-1}$ определено на полуоси $t \geqslant 0$ и стремится $\kappa$ нулю при $t \rightarrow+\infty$.

Для доказательства достаточно заметить, что любое решение системы (4.7) записывается в виде

$$
y_{j}(t)=\ln \frac{\xi_{j+1}(t)}{\xi_{j}(t)}, \quad j=1, \ldots, m-1,
$$


где $\left(\xi_{1}(t), \ldots, \xi_{m}(t)\right)$ - произвольное решение линейной системы

$$
\dot{\xi}_{j}=d\left(\xi_{j+1}-2 \xi_{j}+\xi_{j-1}\right), \quad j=1, \ldots, m, \quad \xi_{0}=\xi_{1}, \quad \xi_{m+1}=\xi_{m},
$$

принадлежащее инвариантному конусу $K=\left\{\left(\xi_{1}, \ldots, \xi_{m}\right): \xi_{j}>0, j=1, \ldots, m\right\}$. А отсюда и из известных свойств системы (4.9) утверждение леммы вытекает очевидным образом.

4.2. Предельный объект. Аналогом предельного отображения (3.78) в данном случае служит $(m-1)$-мерное отображение

$$
\left.z \rightarrow \Phi_{0}(z) \stackrel{\text { def }}{=}\left(y_{1}^{0}(t, z), \ldots, y_{m-1}^{0}(t, z)\right)\right|_{t=T_{0}},
$$

где $z=\left(z_{1}, \ldots, z_{m-1}\right) \in \mathbb{R}^{m-1},\left(y_{1}^{0}(t, z), \ldots, y_{m-1}^{0}(t, z)\right)$ - решение импульсной системы $(4.5)$ на отрезке $0 \leqslant t \leqslant T_{0}$ с начальным условием $\left.\left(y_{1}^{0}, \ldots, y_{m-1}^{0}\right)\right|_{t=0}=z$. Подчеркнем, что в силу явных формул (4.8), (4.9), описывающих поведение упомянутого решения между импульсами, отображение (4.10) заведомо определено во всем пространстве $\mathbb{R}^{m-1}$.

Опираясь на теорему 4.1 о $C^{1}$-сходимости, нетрудно установить аналогичный теореме 3.2 результат о соответствии между гиперболическими неподвижными точками отображения (4.10) и релаксационными циклами исходной системы (4.1). А именно, справедливо следующее утверждение (сохраняющееся, как уже отмечалось ранее, и в случае $\left.a_{0}<1\right)$.

ТЕОРема 4.2. Любой неподвижной точке $z=z_{*}$ отображения (4.10), экспоненциально устойчивой или дихотомичной, в системе (4.1) при всех достаточно малых $\varepsilon>0$ соответствует релаксационный цикл с теми же свойствами устойчивости.

Поиск аттракторов отображения (4.10) начнем с анализа свойств устойчивости его неподвижной точки $z=0$.

ЛЕмма 4.2. Нулевая неподвижная точка отображения (4.10) экспоненииально устойчива при любом $d>0$.

ДокАзАтельство. Несложный подсчет показывает, что отвечающая точке $z=0$ матрица Якоби $\Phi_{0}^{\prime}(0)$ есть оператор сдвига по решениям импульсной системы

$$
\begin{gathered}
\dot{h}_{j}=d\left(h_{j+1}-2 h_{j}+h_{j-1}\right), \\
h_{j}(1+0)=h_{j}(1-0)-\left(1+a_{0}\right) h_{j}(0), \quad j=1, \ldots, m-1, \quad h_{0}=h_{m}=0, \\
h_{j}\left(t_{0}+1+0\right)=h_{j}\left(t_{0}+1-0\right)-\left(1+\frac{1}{a_{0}}\right) h_{j}\left(t_{0}\right)
\end{gathered}
$$

за время от $t=0$ до $t=T_{0}$. Далее, применим к (4.11) метод Фурье по собственным векторам разностного оператора Лапласа, а точнее говоря, положим

$$
h_{j}=\sum_{k=1}^{m-1} g_{k}(t) \sin \left(\frac{\pi k}{m} j\right), \quad j=1, \ldots, m-1 .
$$


В результате убеждаемся, что компоненты $g_{k}(t), k=1, \ldots, m-1$, из (4.12) являются решениями аналогичной (3.83) импульсной системы

$$
\begin{gathered}
\dot{g}=-s g \\
g(1+0)=g(1-0)-\left(1+a_{0}\right) g(0), \\
g\left(t_{0}+1+0\right)=g\left(t_{0}+1-0\right)-\left(1+\frac{1}{a_{0}}\right) g\left(t_{0}\right)
\end{gathered}
$$

при $0 \leqslant t \leqslant T_{0}, s=s_{k}$, где

$$
s_{k}=4 d \sin ^{2}\left(\frac{\pi k}{2 m}\right), \quad k=1, \ldots, m-1 .
$$

На завершающем этапе дополним систему (4.13) начальным условием $\left.g\right|_{t=0}=1$, проинтегрируем получившуюся задачу Коши и положим $\mu_{k}=$ $\left.g\right|_{t=T_{0}, s=s_{k}}$, где $s_{k}$ - дискретные значения (4.14) параметра $s$. В итоге получаем набор чисел $\mu_{k}, k=1, \ldots, m-1$, который, как нетрудно увидеть, образует спектр интересующей нас матрицы $\Phi_{0}^{\prime}(0)$. Более того, справедливы соотношения $\mu_{k}=\left.\mu(s)\right|_{s=s_{k}}, k=1, \ldots, m-1$, где $\mu(s)-$ функция из $(3.84)$, обладающая свойствами (3.85). А отсюда очевидным образом следует, что $\mu_{k} \in(0,1), k=1, \ldots, m-1$. Лемма 4.2 доказана.

В исходной диффузионной цепочке (1.10) неподвижной точке $z=0$ отвечает однородный цикл (1.11). Лемма 4.2 и теорема 4.2 приводят к выводу, что, как и в случае $m=2$, этот цикл является экспоненциально орбитально устойчивым при любом фиксированном значении $d>0$ и при всех достаточно больших $\lambda$.

Отыскание других устойчивых неподвижных точек отображения (4.10) будем проводить в предположении о малости параметра $d$, что позволяет асимптотически проинтегрировать систему (4.5) на отрезке $0 \leqslant t \leqslant T_{0}$. Соответствующий анализ начнем с простейшего случая, когда начальные условия для упомянутой системы заданы аналогичными (3.87) равенствами

$$
\left.y_{j}\right|_{t=0}=\frac{1}{a_{0}} \ln \frac{1}{d}+u_{j}, \quad u_{j}=\text { const } \in \mathbb{R}, \quad j=1, \ldots, m-1,
$$

а параметр $a_{0}$, как и ранее, удовлетворяет условию (3.4).

Зафиксируем произвольно компактное множество $\Omega \subset \mathbb{R}^{m-1}$ и обозначим через

$$
\left(y_{1}(t, u, d), \ldots, y_{m-1}(t, u, d)\right), \quad 0 \leqslant t \leqslant T_{0}, \quad u=\left(u_{1}, \ldots, u_{m-1}\right) \in \Omega,
$$

решение задачи Коши (4.5), (4.15). Далее, действуя таким же образом, как и при выводе отображения (3.99), для компонент $y_{j}(t, u, d), j=1, \ldots, m-1$, из (4.16) получаем асимптотические равенства

$$
\begin{array}{ll}
y_{j}(t, u, d)=\frac{1}{a_{0}} \ln \frac{1}{d}+u_{j}+O\left(d^{1-1 / a_{0}}\right) & \text { при } t \in[0,1), \\
y_{j}(t, u, d)=-\ln \frac{1}{d}+h_{j}^{0}(t, u)+O\left(d^{1-1 / a_{0}}\right) & \text { при } t \in\left[1, t_{0}+1\right), \\
y_{j}(t, u, d)=\frac{1}{a_{0}} \ln \frac{1}{d}+\psi_{j}(u)+O\left(d^{1-1 / a_{0}}\right) & \text { при } t \in\left[t_{0}+1, T_{0}\right],
\end{array}
$$


где $\left(h_{1}^{0}(t, u), \ldots, h_{m-1}^{0}(t, u)\right)$ - решение задачи Коши

$$
\begin{aligned}
\dot{h}_{1}=\exp \left(-h_{1}\right), \quad \dot{h}_{j} & =\exp \left(-h_{j}\right)-\exp \left(-h_{j-1}\right), \quad j=2, \ldots, m-1, \\
\left.h_{j}\right|_{t=1} & =-a_{0} u_{j}, \quad j=1, \ldots, m-1,
\end{aligned}
$$

а функции $\psi_{j}(u), j=1, \ldots, m-1$, задаются формулами

$$
\psi_{j}(u)=\left.h_{j}^{0}(t, u)\right|_{t=t_{0}+1}-\left.\left(1+\frac{1}{a_{0}}\right) h_{j}^{0}(t, u)\right|_{t=t_{0}}, \quad j=1, \ldots, m-1 .
$$

Добавим еще, что как сами равенства (4.17)-(4.19), так и аналогичные асимптотические представления, получающиеся из них в результате дифференцирования по $u$, выполняются равномерно по $t$ из соответствующих промежутков и по $u \in \Omega$. Принимая во внимание указанные свойства, нетрудно убедиться, что после перехода к переменным $u_{j}=z_{j}-\left(1 / a_{0}\right) \ln (1 / d), j=1, \ldots, m-1$, интересующее нас отображение (4.10) записывается в виде

$$
u_{j} \rightarrow \psi_{j}(u)+\Delta_{j}(u, d), \quad j=1, \ldots, m-1,
$$

где остатки $\Delta_{j}(u, d)$ имеют порядок малости $O\left(d^{1-1 / a_{0}}\right)$ в метрике пространства $C^{1}(\Omega)$. А это значит, что при изучении вопроса о неподвижных точках мы вправе заменить (4.22) предельным отображением

$$
u_{j} \rightarrow \psi_{j}(u), \quad j=1, \ldots, m-1 .
$$

Исследование отображения (4.23) существенно опирается на тот факт, что компоненты $h_{j}^{0}(t, u)$ решения задачи (4.20) могут быть найдены в явном виде. Действительно, непосредственная проверка показывает, что для них справедливы равенства

$$
\begin{gathered}
h_{1}^{0}(t, u)+\cdots+h_{k}^{0}(t, u)=\ln \left\{\frac{(t-1)^{k}}{k !}+\sum_{\ell=0}^{k-1} \frac{(t-1)^{\ell}}{\ell !} \exp \left(-a_{0} \sum_{j=1}^{k-\ell} u_{j}\right)\right\}, \\
k=1, \ldots, m-1 .
\end{gathered}
$$

Далее, подставим (4.24) в (4.21) и перейдем к новым переменным

$$
\alpha_{k}=u_{1}+\cdots+u_{k}, \quad k=1, \ldots, m-1 .
$$

В результате наше отображение примет треугольный вид

$$
\begin{array}{r}
\alpha_{k} \rightarrow \ln \left(r_{1, k}+\exp \left(-a_{0} \alpha_{k}\right)\right)-\left(1+\frac{1}{a_{0}}\right) \ln \left(r_{2, k}+\exp \left(-a_{0} \alpha_{k}\right)\right), \\
k=1, \ldots, m-1,
\end{array}
$$

где

$$
\begin{gathered}
r_{1,1}=1+\frac{1}{a_{0}}, \quad r_{2,1}=\frac{1}{a_{0}}, \\
r_{1, k}\left(\alpha_{1}, \ldots, \alpha_{k-1}\right)=\frac{\left(1+1 / a_{0}\right)^{k}}{k !}+\sum_{\ell=1}^{k-1} \frac{\left(1+1 / a_{0}\right)^{\ell}}{\ell !} \exp \left(-a_{0} \alpha_{k-\ell}\right), \\
r_{2, k}\left(\alpha_{1}, \ldots, \alpha_{k-1}\right)=\frac{1}{a_{0}^{k} k !}+\sum_{\ell=1}^{k-1} \frac{1}{a_{0}^{\ell} \ell !} \exp \left(-a_{0} \alpha_{k-\ell}\right), \quad k=2, \ldots, m-1 .
\end{gathered}
$$


Анализ получившегося отображения (4.26) достаточно прост. В самом деле, от него очевидным образом "отщепляется" одномерное отображение для координаты $\alpha_{1}$, имеющее вид

$$
\left.\alpha_{1} \rightarrow \psi_{r_{1}, r_{2}}\left(\alpha_{1}\right)\right|_{r_{1}=r_{1,1}, r_{2}=r_{2,1}}
$$

где $\psi_{r_{1}, r_{2}}$ - отображение (3.100). Кроме того, параметры $r_{1,1}, r_{2,1}$, задаваемые равенствами (4.27), удовлетворяют условию (3.101) (это условие эквивалентно требованию $a_{0}>1$, которое мы a priori считаем выполненным). А отсюда и из леммы 3.3 вытекает существование у (4.29) единственной экспоненциально устойчивой неподвижной точки $\alpha_{1}=\alpha_{1}^{*}$.

Предположим теперь, что уже найдены первые $k-1$ координат $\alpha_{1}^{*}, \ldots, \alpha_{k-1}^{*}$ устойчивой неподвижной точки отображения (4.26). Тогда после подстановки в (4.28) известных значений $\alpha_{s}=\alpha_{s}^{*}, s=1, \ldots, k-1$, для определения $\alpha_{k}$ получаем аналогичное (4.29) одномерное отображение

$$
\left.\alpha_{k} \rightarrow \psi_{r_{1}, r_{2}}\left(\alpha_{k}\right)\right|_{r_{1}=r_{1, k}^{*}, r_{2}=r_{2, k}^{*}},
$$

где $r_{1, k}^{*}=r_{1, k}\left(\alpha_{1}^{*}, \ldots, \alpha_{k-1}^{*}\right), r_{2, k}^{*}=r_{2, k}\left(\alpha_{1}^{*}, \ldots, \alpha_{k-1}^{*}\right)$. Заметим, далее, что и здесь условие (3.101) остается в силе, поскольку

$$
\begin{aligned}
r_{1, k}^{*}-\left(1+\frac{1}{a_{0}}\right) r_{2, k}^{*}=( & \left.+\frac{1}{a_{0}}\right)\left\{\frac{1}{k !}\left(\left(1+\frac{1}{a_{0}}\right)^{k-1}-\frac{1}{a_{0}^{k}}\right)\right. \\
& \left.+\sum_{\ell=1}^{k-1} \frac{1}{\ell !}\left(\left(1+\frac{1}{a_{0}}\right)^{\ell-1}-\frac{1}{a_{0}^{\ell}}\right) \exp \left(-a_{0} \alpha_{k-\ell}^{*}\right)\right\}
\end{aligned}
$$

и $\left(1+1 / a_{0}\right)^{\ell-1}>1 / a_{0}^{\ell}$ для любого $\ell \leqslant k$. А это означает, что отображение $(4.30)$ имеет единственную экспоненциально устойчивую неподвижную точку $\alpha_{k}=\alpha_{k}^{*}$.

Продолжая описанный процесс вплоть до номера $k=m-1$, убеждаемся, что исходное отображение (4.26) допускает единственную экспоненциально устойчивую неподвижную точку с компонентами $\left(\alpha_{1}^{*}, \ldots, \alpha_{m-1}^{*}\right)$. Далее, обращая замену (4.25), получаем устойчивую неподвижную точку $\left(u_{1}^{*}, \ldots, u_{m-1}^{*}\right)$ отображения (4.23) и соответствующую близкую к ней неподвижную точку $\left(u_{1}(d), \ldots, u_{m-1}(d)\right): u_{j}(0)=u_{j}^{*}, j=1, \ldots, m-1$ отображения (4.22). Что же касается отображения (4.10), то координаты его устойчивой неподвижной точки задаются равенствами

$$
z_{j}=\frac{1}{a_{0}} \ln \frac{1}{d}+u_{j}(d), \quad j=1, \ldots, m-1 .
$$

Следует добавить, что неподвижная точка (4.31) существует в паре с устойчивой неподвижной точкой

$$
z_{j}=-\frac{1}{a_{0}} \ln \frac{1}{d}-u_{m-j}(d), \quad j=1, \ldots, m-1 .
$$

Наличие последней обусловлено тем обстоятельством, что отображение (4.10) инвариантно по отношению к замене

$$
z_{j} \rightarrow-z_{m-j}, \quad j=1, \ldots, m-1 .
$$


Отметим еще, что неподвижным точкам (4.31), (4.32) в диффузионной цепочке (1.10) отвечает пара устойчивых релаксационных циклов, переходящих друг в друга при замене

$$
N_{j} \rightarrow N_{m+1-j}, \quad j=1, \ldots, m .
$$

Завершая обсуждение свойств отображения (4.10), отметим, что помимо найденных выше трех устойчивых неподвижных точек у него имеются и другие аттракторы. Для отыскания части из них зафиксируем натуральное $k_{0}$, $k_{0} \leqslant m-2$, и дополним систему (4.5) аналогичными (4.15) начальными условиями

$$
\begin{array}{ll}
\left.y_{j}\right|_{t=0}=-\frac{1}{a_{0}} \ln \frac{1}{d}+u_{j}, & j=1, \ldots, k_{0}, \\
\left.y_{j}\right|_{t=0}=\frac{1}{a_{0}} \ln \frac{1}{d}+u_{j}, & j=k_{0}+1, \ldots, m-1,
\end{array}
$$

где по-прежнему параметр $d$ мал, $u_{j}=$ const $\in \mathbb{R}, j=1, \ldots, m-1$, а постоянная $a_{0}$ удовлетворяет оценке (3.4). В результате для компонент $y_{j}(t, u, d)$, $j=1, \ldots, m-1$, решения получившейся задачи Коши (4.5), (4.33) приходим к аналогичным (4.17)-(4.19) асимптотическим равенствам:

$$
\begin{array}{ll}
y_{j}=-\frac{1}{a_{0}} \ln \frac{1}{d}+u_{j}+O\left(d^{1-1 / a_{0}}\right), \quad j=1, \ldots, k_{0}, & \text { при } t \in[0,1) ; \\
y_{j}=\frac{1}{a_{0}} \ln \frac{1}{d}+u_{j}+O\left(d^{1-1 / a_{0}}\right), \quad j=k_{0}+1, \ldots, m-1, & \\
y_{j}=\ln \frac{1}{d}+h_{j}^{0}(t, u)+O\left(d^{1-1 / a_{0}}\right), \quad j=1, \ldots, k_{0}, & \text { при } t \in\left[1, t_{0}+1\right) ; \\
y_{j}=-\ln \frac{1}{d}+h_{j}^{0}(t, u)+O\left(d^{1-1 / a_{0}}\right), \quad j=k_{0}+1, \ldots, m-1, & \\
y_{j}=-\frac{1}{a_{0}} \ln \frac{1}{d}+\psi_{j}(u)+O\left(d^{1-1 / a_{0}}\right), & j=1, \ldots, k_{0}, \\
y_{j}=\frac{1}{a_{0}} \ln \frac{1}{d}+\psi_{j}(u)+O\left(d^{1-1 / a_{0}}\right), & j=k_{0}+1, \ldots, m-1,
\end{array}
$$

где $\left(h_{1}^{0}(t, u), \ldots, h_{m-1}^{0}(t, u)\right)$ - решение задачи Коши

$$
\begin{gathered}
\dot{h}_{j}=\exp h_{j+1}-\exp h_{j}, \quad j=1, \ldots, k_{0}-1, \\
\dot{h}_{k_{0}}=-\exp h_{k_{0}}, \\
\left.h_{j}\right|_{t=1}=-a_{0} u_{j}, \quad j=1, \ldots, k_{0} ; \\
\dot{h}_{k_{0}+1}=\exp \left(-h_{k_{0}+1}\right), \\
\dot{h}_{j}=\exp \left(-h_{j}\right)-\exp \left(-h_{j-1}\right), \quad j=k_{0}+2, \ldots, m-1, \\
\left.h_{j}\right|_{t=1}=-a_{0} u_{j}, \quad j=k_{0}+1, \ldots, m-1,
\end{gathered}
$$

а для функций $\psi_{j}(u), j=1, \ldots, m-1$, сохраняются формулы (4.32). 
Дальнейший способ действий аналогичен изложенному выше: после подстановки в (4.10) соотношений

$$
\begin{array}{rlrl}
z_{j} & =-\frac{1}{a_{0}} \ln \frac{1}{d}+u_{j}, & & j=1, \ldots, k_{0}, \\
z_{j}=\frac{1}{a_{0}} \ln \frac{1}{d}+u_{j}, & j=k_{0}+1, \ldots, m-1,
\end{array}
$$

и предельного перехода при $d \rightarrow 0$ (законность которого в метрике пространства $C^{1}(\Omega)$ вытекает из формул (4.34)-(4.36)) получаем соответствующее отображение для $u=\left(u_{1}, \ldots, u_{m-1}\right)$. Специфика рассматриваемого случая заключается в том, что в силу независимости подсистем (4.37) и (4.38) упомянутое отображение также распадается на два независимых отображения

$$
\begin{array}{ll}
u_{j} \rightarrow \psi_{j}\left(u_{1}, \ldots, u_{k_{0}}\right), & j=1, \ldots, k_{0} ; \\
u_{j} \rightarrow \psi_{j}\left(u_{k_{0}+1}, \ldots, u_{m-1}\right), & j=k_{0}+1, \ldots, m-1 .
\end{array}
$$

Сравнивая системы (4.20) и (4.38), нетрудно увидеть, что после надлежащего переобозначения координат отображение (4.40) в точности совпадет с отображением (4.23) (при замене в последнем $m$ на $m-k_{0}$ ). Что же касается отображения (4.39), то оно приводится к (4.23) преобразованиями $u_{j} \rightarrow-u_{k_{0}+1-j}$, $j=1, \ldots, k_{0}$. А отсюда и из проделанного выше анализа вытекает, что полное отображение (4.39), (4.40) имеет единственную экспоненциально устойчивую неподвижную точку $\left(u_{1}^{*}, \ldots, u_{m-1}^{*}\right)$.

Суммируя проделанные построения и меняя $k_{0}$ в пределах от единицы до $m-2$, приходим к выводу, что при всех достаточно малых $d>0$ и при условии (3.4) отображение (4.10) допускает набор из $m-2$ устойчивых неподвижных точек с координатами

$$
\begin{array}{ll}
z_{j}=-\frac{1}{a_{0}} \ln \frac{1}{d}+u_{j}^{*}+O\left(d^{1-1 / a_{0}}\right), & j=1, \ldots, k_{0}, \\
z_{j}=\frac{1}{a_{0}} \ln \frac{1}{d}+u_{j}^{*}+O\left(d^{1-1 / a_{0}}\right), & j=k_{0}+1, \ldots, m-1,
\end{array}
$$

где $u_{j}^{*}, j=1, \ldots, m-1,-$ компоненты неподвижной точки предельного отображения (4.39), (4.40). Общее же количество найденных нами устойчивых ненулевых неподвижных точек отображения (4.10) равно $m$ (см. (4.31), (4.32), (4.41)).

4.3. Заключение. Теорема 4.2 о соответствии и содержащийся в предыдущем пункте асимптотический анализ отображения (4.10) приводят к следующему утверждению, являющемуся основным результатом данной статьи.

Теорема 4.3. Пусть параметр $a_{0}$ в (1.6) удовлетворяет условию (3.4). Тогда для любых достаточно малых $d_{2}>d_{1}>0$ найдется $\lambda_{0}=\lambda_{0}\left(d_{1}, d_{2}\right)>0$ такое, что при всех $d_{1} \leqslant d \leqslant d_{2}, \lambda \geqslant \lambda_{0}$ система (1.10) имеет не менее $m$ экспоненииально орбитально устойчивых пространственно неоднородных ииклов, сосуществующих с устойчивым ииклом (1.11).

В случае $a_{0}<1$ утверждение теоремы 4.3 сохраняется по крайней мере при $m=2$. Связано это с тем, что здесь после подстановки в (3.79) соотношения $z=$ 
$\ln (1 / d)+u, u=$ const $\in \mathbb{R}$, и асимптотического интегрирования получившейся задачи Коши выходит аналогичное (3.99) отображение

$$
u \rightarrow \psi(u) \stackrel{\text { def }}{=}-\ln \left[a_{0}+(1+\exp (-u))^{-1 / a_{0}} \exp \left(-\left(1+\frac{1}{a_{0}}\right) u\right)\right],
$$

имеющее единственную глобально экспоненциально устойчивую неподвижную точку $u=u_{*}$. Далее, возвращаясь к исходному отображению (3.78), заключаем, что при $a_{0}<1$ оно по-прежнему допускает две устойчивые неподвижные точки $z= \pm z(d)$, где

$$
z(d)=\ln \frac{1}{d}+u_{*}+O\left(d^{1-a_{0}}\right), \quad d \rightarrow 0 .
$$

Вопрос о других аттракторах отображения (4.10), отличных от найденных в п. 4.2 неподвижных точек, пока остается открытым. Для пояснения возникающих здесь трудностей, считая выполненным неравенство (3.4), дополним систему (4.5) начальными условиями вида

$$
\left.y_{j}\right|_{t=0}= \pm \frac{1}{a_{0}} \ln \frac{1}{d}+u_{j}, \quad u_{j}=\text { const } \in \mathbb{R}, \quad j=1, \ldots, m-1,
$$

при произвольной расстановке знаков "+" и "-_. Несложный подсчет показывает, что асимптотическое интегрирование задачи Коши (4.5), (4.42) приводит к некоторому предельному $(m-1)$-мерному отображению для $u=\left(u_{1}, \ldots, u_{m-1}\right)$. Таким образом, исходное отображение (4.10) при $d \rightarrow 0$ порождает $2^{m-1}$ различных предельных отображений. Однако отнюдь не все эти отображения обладают гиперболическими аттракторами.

Например, при $m=3$ и при выборе в $(4.42)$ порядка знаков $(+,-)$ получается отображение

$$
\left.\alpha_{1} \rightarrow \psi_{r_{1}, r_{2}}\left(\alpha_{1}\right)\right|_{r_{1}=r_{1}\left(\alpha_{2}\right), r_{2}=r_{2}\left(\alpha_{2}\right)}, \quad \alpha_{2} \rightarrow \alpha_{2},
$$

где $\alpha_{1}=u_{1}, \alpha_{2}=u_{1}+u_{2}, \psi_{r_{1}, r_{2}}$ - отображение $(3.100)$, а функции $r_{1}\left(\alpha_{2}\right)$, $r_{2}\left(\alpha_{2}\right)$ задаются соотношениями

$$
r_{1}\left(\alpha_{2}\right)=\left(1+\frac{1}{a_{0}}\right)\left(1+\exp \left(-a_{0} \alpha_{2}\right)\right), \quad r_{2}\left(\alpha_{2}\right)=\frac{1}{a_{0}}\left(1+\exp \left(-a_{0} \alpha_{2}\right)\right) .
$$

Заметим, далее, что в силу вытекающего из (4.44) очевидного неравенства $r_{1}\left(\alpha_{2}\right)>\left(1+1 / a_{0}\right) r_{2}\left(\alpha_{2}\right) \forall \alpha_{2} \in \mathbb{R}$ и леммы 3.3 отображение (4.43) допускает устойчивый однопараметрический континуум неподвижных точек

$$
\left(\alpha_{1}, \alpha_{2}\right): \alpha_{1}=\left.u_{*}\left(r_{1}, r_{2}\right)\right|_{r_{1}=r_{1}\left(\alpha_{2}\right), r_{2}=r_{2}\left(\alpha_{2}\right)}, \quad \alpha_{2} \in \mathbb{R},
$$

где $u_{*}\left(r_{1}, r_{2}\right)$ - неподвижная точка модельного отображения (3.100).

Переход от отображения (4.43) к исходному отображению (4.10) связан с возмущением правых частей из (4.43) добавками порядка малости $O\left(d^{1-1 / a_{0}}\right)$ (см., например, (3.97) и (4.22)). Вопрос же о том, какие из неподвижных точек (4.45) при этом выживут и каковыми будут их свойства устойчивости, подлежит отдельному рассмотрению, выходящему за рамки данной статьи. 
Отметим, что с континуумом неподвижных точек в предельном отображении мы сталкиваемся во всех ситуациях, когда в последовательности знаков "+" и "-" из (4.42) имеется фрагмент $(+,-)$. Таким образом, стала понятной причина, по которой мы в п. 4.2 ограничились рассмотрением лишь случаев (4.31), (4.32) и (4.41).

Остановимся на еще одной нерешенной проблеме. Следуя принятой в книгах [14], [15] терминологии, ситуацию, которая реализуется в рамках теоремы 4.3, будем называть диффузионной буферностью. Далее, поставим вопрос о том, сохранится ли упомянутый феномен при переходе от дискретных цепочек (1.10) к соответствующей распределенной модели, т.е. при $d=m^{2} D$, $D=$ const $>0$ и при $m \rightarrow \infty$.

В математическом плане сформулированная проблема сводится к исследованию аттракторов импульсной системы

$$
\begin{gathered}
\dot{y}_{j}=m^{2} D\left[\exp y_{j+1}+\exp \left(-y_{j}\right)-\exp y_{j}-\exp \left(-y_{j-1}\right)\right], \\
y_{j}\left(t_{2 k-1}+0\right)=y_{j}\left(t_{2 k-1}-0\right)-\left(1+a_{0}\right) y_{j}\left(t_{2 k-1}-1\right), \\
y_{j}\left(t_{2 k}+0\right)=y_{j}\left(t_{2 k}-0\right)-\left(1+\frac{1}{a_{0}}\right) y_{j}\left(t_{2 k}-1\right), \\
k \in \mathbb{N}, \quad j=1, \ldots, m-1, \quad y_{0}=y_{m}=0,
\end{gathered}
$$

где моменты скачков $t_{2 k-1}, t_{2 k}$ те же самые, что и в (3.73). Точнее говоря, интересующий нас вопрос звучит так: сохраняются ли в системе (4.46) устойчивые автоволновые процессы при фиксированном достаточно малом $D>0$ и при $m \rightarrow \infty$ ? Помимо этого представляет интерес анализ возможных аттракторов импульсной краевой задачи

$$
\begin{aligned}
\frac{\partial v}{\partial t} & =D\left(\frac{\partial^{2} v}{\partial x^{2}}+2 v \frac{\partial v}{\partial x}\right),\left.\quad v\right|_{x=0}=\left.v\right|_{x=1}=0, & & \\
v\left(t_{2 k-1}+0, x\right) & =v\left(t_{2 k-1}-0, x\right)-\left(1+a_{0}\right) v\left(t_{2 k-1}-1, x\right), & & k \in \mathbb{N}, \\
v\left(t_{2 k}+0, x\right) & =v\left(t_{2 k}-0, x\right)-\left(1+\frac{1}{a_{0}}\right) v\left(t_{2 k}-1, x\right), & & k \in \mathbb{N},
\end{aligned}
$$

на отрезке $0 \leqslant x \leqslant 1$, получающейся из (4.46) при $y_{j}=v_{j} / m$ и при $m \rightarrow \infty$.

Следует добавить, что как в системе (4.46), так и в рамках краевой задачи (4.47) (рассматриваемой в фазовом пространстве $\stackrel{\circ}{W} \underset{2}{1}(0,1))$ нулевое решение является экспоненциально устойчивым при любом $D>0$. Тем самым, при изучении поставленных выше вопросов речь должна идти только о ненулевых аттракторах. Ясно также, что отыскание последних возможно лишь с использованием численных методов.

Представляет интерес выявление биологических причин, по которым в цепочке (1.10) реализуется феномен диффузионной буферности. Казалось бы, наиболее естественным ее аттрактором должен быть однородный цикл (1.11). Однако, как свидетельствуют численные эксперименты, даже в простейшем случае $m=2$ область притяжения упомянутого цикла при достаточно малых 
значениях параметра $d$ весьма узка. С вычислительной точки зрения это означает, что при наугад выбранных начальных условиях в системе (1.10) с вероятностью, близкой к единице, будет наблюдаться не цикл (1.11), а некоторый устойчивый пространственно неоднородный периодический режим.

Для того чтобы разобраться в причинах такого поведения траекторий, введем следующие определения. Каждому устойчивому периодическому решению $\left(N_{1}(t), \ldots, N_{m}(t)\right)$ системы (1.10) периода $T>0$ поставим в соответствие его динамическое среднее значение

$$
\mathscr{U}\left[\left(N_{1}, \ldots, N_{m}\right)\right]=\frac{1}{T} \int_{0}^{T}\left(\frac{1}{m} \sum_{j=1}^{m} N_{j}(t)\right) d t .
$$

Заметим, далее, что для всех найденных нами устойчивых циклов величина (4.48) асимптотически велика (точнее говоря, имеет порядок $(\exp \lambda) / \lambda$ ). В связи с этим для характеристики того или иного периодического режима вместо (4.48) удобнее использовать функционал

$$
U\left[\left(N_{1}, \ldots, N_{m}\right)\right]=\frac{\mathscr{U}\left[\left(N_{1}, \ldots, N_{m}\right)\right]}{\mathscr{U}\left[\left(N_{*}, \ldots, N_{*}\right)\right]},
$$

где, напомним, $\left(N_{*}, \ldots, N_{*}\right)$ - однородный цикл (1.11).

Введенный выше функционал (4.49) позволяет сравнивать между собой различные циклы системы (1.10). А именно, будем говорить, что периодическое решение $\left(N_{1}^{(1)}(t), \ldots, N_{m}^{(1)}(t)\right)$ биологически предпочтительнее другого периодического решения $\left(N_{1}^{(2)}(t), \ldots, N_{m}^{(2)}(t)\right)$, если

$$
U\left[\left(N_{1}^{(1)}, \ldots, N_{m}^{(1)}\right)\right]>U\left[\left(N_{1}^{(2)}, \ldots, N_{m}^{(2)}\right)\right] .
$$

Естественно ожидать, что наибольшие области притяжения имеют именно те периодические режимы, на которых функционал (4.49) максимален или близок к таковому. Для проверки этого факта обратимся к случаю $m=2$. Как следует из полученных нами результатов, соответствующая система (3.1) при фиксированном достаточно малом $d>0$, при $a_{0} \neq 1$ и при всех $\lambda \gg 1$ допускает три аттрактора: однородный цикл (3.86) и два устойчивых неоднородных цикла $\left(N_{1}(t, \lambda, d), N_{2}(t, \lambda, d)\right),\left(N_{2}(t, \lambda, d), N_{1}(t, \lambda, d)\right)$ периода $T(\lambda, d)$, отвечающих двум устойчивым ненулевым неподвижным точкам отображения (3.78). Далее, положим

$$
U(\lambda, d)=U\left[\left(N_{1}(t, \lambda, d), N_{2}(t, \lambda, d)\right)\right]=U\left[\left(N_{2}(t, \lambda, d), N_{1}(t, \lambda, d)\right)\right] .
$$

Справедливо следующее утверждение.

ЛЕмма 4.3. При $\lambda \rightarrow \infty$ функиия (4.50) стремится $\kappa$ конечному пределу

$$
U_{0}(d)=\frac{\operatorname{ch}\left(\gamma_{1} / 2\right)}{\operatorname{ch}\left(\gamma_{2} / 2\right)} \operatorname{ch}\left[\frac{\gamma_{1}-\gamma_{2}}{2}\right]>1
$$

где $\gamma_{1}=\left.y_{0}(0, z)\right|_{z=z(d)}, \gamma_{2}=\left.y_{0}(1-0, z)\right|_{z=z(d)}, y_{0}(t, z)$ - решение задачи Коши (3.79), а $z=z(d)>0$ - устойчивая неподвижная точка отображения (3.78). 
ДокАЗАТЕЛЬство. Проведем сначала асимптотическое вычисление динамического среднего значения (4.48) для однородного цикла. Обращаясь к равенствам (2.58), (2.59) и асимптотическим формулам (2.14), (2.17), (2.32), (2.33), (2.44), нетрудно увидеть, что

$$
\begin{gathered}
\int_{0}^{1-\varepsilon^{\delta}} N_{*}(t, \lambda) d t+\int_{1+\varepsilon^{\delta}}^{T_{*}} N_{*}(t, \lambda) d t \leqslant M \exp \left[\frac{1-\min \left(1, a_{0}\right) \varepsilon^{\delta}}{\varepsilon}\right], \\
\int_{1-\varepsilon^{\delta}}^{1+\varepsilon^{\delta}} N_{*}(t, \lambda) d t=\varepsilon \exp \left(\frac{1}{\varepsilon}\right)\left(\int_{-\infty}^{+\infty} \exp [v(\tau)] d \tau+o(1)\right),
\end{gathered}
$$

где $\varepsilon=1 / \lambda, \delta$ - произвольная фиксированная постоянная из интервала $(0,1)$, $M>0$ - некоторая не зависящая от $\varepsilon$ константа, $v(\tau)-$ функция (2.18). А отсюда очевидным образом следует, что

$$
\mathscr{U}\left[\left(N_{*}, N_{*}\right)\right]=\frac{\varepsilon \exp (1 / \varepsilon)}{T_{0}}\left(\int_{-\infty}^{+\infty} \exp [v(\tau)] d \tau+o(1)\right) .
$$

В случае пространственно неоднородного цикла $\left(N_{1}(t, \lambda, d), N_{2}(t, \lambda, d)\right)$ рассуждения аналогичны. Опираясь на соответствующие асимптотические формулы из п. 3.2 (см. (3.24), (3.26), (3.46), (3.47), (3.49)-(3.51), (3.59), (3.60), (3.62), (3.63)), убеждаемся, что для обеих компонент $N_{1}, N_{2}$ упомянутого цикла неравенства вида (4.52) сохраняются. Что же касается интегралов по отрезку $1-\varepsilon^{\delta} \leqslant t \leqslant 1+\varepsilon^{\delta}$, то для них с учетом асимптотических представлений (3.46) получаем аналогичные (4.53) формулы

$$
\begin{aligned}
\int_{1-\varepsilon^{\delta}}^{1+\varepsilon^{\delta}} N_{1}(t, \lambda, d) d t & =\varepsilon \exp \left(\frac{1}{\varepsilon}\right)\left(\int_{-\infty}^{+\infty} \exp \left[\left.v_{1}^{0}(\tau, z)\right|_{z=\tilde{z}(d)}\right] d \tau+o(1)\right) \\
\int_{1-\varepsilon^{\delta}}^{1+\varepsilon^{\delta}} N_{2}(t, \lambda, d) d t & =\varepsilon \exp \left(\frac{1}{\varepsilon}\right)\left(\int_{-\infty}^{+\infty} \exp \left[\left.\left(v_{1}^{0}(\tau, z)+v_{2}^{0}(\tau, z)\right)\right|_{z=\tilde{z}(d)}\right] d \tau+o(1)\right) .
\end{aligned}
$$

Здесь $v_{1}^{0}(\tau, z), v_{2}^{0}(\tau, z)$ - функции $(3.33)$, а $z=\tilde{z}(d)>0$ - устойчивая неподвижная точка отображения (3.22).

Объединяя соотношения $(4.54),(4.55)$ и учитывая, что период рассматриваемого неоднородного цикла в силу (3.64) допускает асимптотику вида $T(\lambda, d)=$ $T_{0}+O(1 / \lambda)$, приходим к выводу о существовании требуемого предела $U_{0}(d)$ функции (4.50) при $\lambda \rightarrow \infty$. Более того, несложный подсчет показывает, что

$$
U_{0}(d)=\left.\frac{1}{2}\left[\exp \varkappa_{3}(z)+\exp \left(\varkappa_{3}(z)+\varkappa_{4}(z)\right)\right]\right|_{z=\tilde{z}(d)},
$$

где $\varkappa_{3}(z), \varkappa_{4}(z)-$ функции (3.34).

На заключительном этапе обоснования перепишем в инвариантной форме правую часть равенства (4.56). Проблема здесь заключается в том, что фигурирующее в (3.34) решение $y_{0}(t, z)$ задачи Коши $(3.11),(3.12)$ зависит от выбора начального момента времени $t=-\sigma_{0}$. Таким образом, от $\sigma_{0}$ должна, в принципе, зависеть и функция $U_{0}(d)$. Однако на самом деле это не так. Действительно, неподвижные точки $z(d)$ и $\tilde{z}(d)$ отображений (3.78) и (3.22) 
связаны соотношением $z(d)=P^{\sigma_{0}}(\tilde{z}(d))$, где $P^{t}$ - оператор сдвига (3.75). Учитывая данное обстоятельство в (4.56) и выполняя в интеграле из (3.34) замену $d s=-d y /(2 d \operatorname{sh} y)$, после некоторых преобразований приходим к инвариантной (не зависящей от $\sigma_{0}$ ) формуле (4.51). Остается добавить, что свойства $y_{0}(t, z)>0, \dot{y}_{0}(t, z)<0 \forall t \in[0,1) \forall z>0$ решения задачи Коши (3.79) гарантируют выполнение неравенств $\gamma_{1}>\gamma_{2}>0$, из которых, в свою очередь, вытекает требуемая оценка $U_{0}(d)>1$. Лемма 4.3 доказана.

Проделанный анализ позволяет утверждать, что два устойчивых пространственно неоднородных цикла системы (3.1) биологически предпочтительнее однородного цикла (3.86) и вследствие этого имеют бо́льшие, чем у него, бассейны притяжения. Таким образом, с биологической точки зрения можно говорить об элементах самоорганизации: рассматриваемый вид искусственно (за счет миграций) создает себе неоднородную среду обитания и повышает свой “уровень жизни", характеризующийся значением функционала (4.49). Устойчивые же периодические режимы, для которых $U>1$, в связи с вышесказанным уместно назвать режимами самоорганизации.

Случай $m>2$ отличается от $m=2$ лишь тем, что различных режимов самоорганизации становится много (не менее $m$ ). Здесь при произвольно заданных начальных условиях происходит конкуренция между устойчивыми циклами и в конечном итоге система (1.10) "выходит" на тот из них, для которого выбранные начальные условия оказались предпочтительнее. Таким образом, эффекты самоорганизации в цепочке (1.10) реализуются через феномен буферности.

В заключение отметим, что предпринятые в данной статье математические исследования были стимулированы компьютерным анализом системы (1.10) при $m=2$ и 3 . Иными словами, численный эксперимент помог создать адекватную асимптотическую теорию.

\section{Список литературы}

[1] Е.Ф. Мищенко, Л.С. Понтрягин, "Периодические решения систем дифференциальных уравнений, близкие к разрывным", Докл. АН CCCP, 102:5 (1955), 889-891.

[2] Е.Ф. Мищенко, "Асимптотическое вычисление периодических решений систем дифференциальных уравнений, содержащих малые параметры при производных", Изв. АН СССР. Сер. матем., 21:5 (1957), 627-654.

[3] Е.Ф. Мищенко, Л. С. Понтрягин, "Доказательство некоторых асимптотических формул для решений дифференциальных уравнений с малым параметром”, Докл. АН СССР, 120:5 (1958), 967-969.

[4] Е. Ф. Мищенко, Л. С. Понтрягин, "Вывод некоторых асимптотических оценок для решений дифференциальных уравнений с малым параметром при производных", Изв. АН СССР. Сер. матем., 23:5 (1959), 643-660.

[5] Л. С. Понтрягин, "Асимптотическое поведение решений систем дифференциальных уравнений с малым параметром при высших производных", Изв. АН СCCP. Сер. матем., 21:5 (1957), 605-626.

[6] Е. Ф. Мищенко, Н. Х. Розов, Дифференциалъные уравнения с малым параметром и релаксационные колебания, Наука, М., 1975, 247 с.; англ. пер.: Е. F. Mishchenko, N. Kh. Rozov, Differential equations with small parameters and relaxation oscillations, 
Math. Concepts Methods Sci. Engrg., 13, Plenum Press, New York, London, 1980, $\mathrm{x}+228 \mathrm{pp}$.

[7] Е. Ф. Мищенко, Ю. С. Колесов, А. Ю. Колесов, Н. Х. Розов, Периодические движения и бифуркационные проц,ссы в сингулярно возмущенных системах, Наука, Физматлит, М., 1995, 336 с.

[8] А. Ю. Колесов, Ю.С. Колесов, Релаксационные колебания в математических моделях экологии, Тр. МИАН, 199, Наука, М., 1993, 126 с.; англ. пер.: A. Yu. Kolesov, Yu. S. Kolesov, "Relaxation oscillations in mathematical models of ecology", Proc. Steklov Inst. Math., 199 (1995), 1-126.

[9] А.Ю. Колесов, Е.Ф. Мищенко, Н.Х. Розов, "Реле с запаздыванием и его $C^{1}$-аппроксимация", Динамические системы и смежные вопросы, Сборник статей. K 60-летию со дня рождения академика Дмитрия Викторовича Аносова, Тр. МИАН, 216, Наука, М., 1997, 126-153; англ. пер.: А. Yu. Kolesov, E.F. Mishchenko, N. Kh. Rozov, "A relay with delay and its $C^{1}$-approximation", Proc. Steklov Inst. Math., 216 (1997), 119-146.

[10] G. E. Hutchinson, "Circular causal systems in ecology", Ann. N. Y. Acad. Sci., 50 (1948), 221-246.

[11] E. M. Wright, "A non-linear difference-differential equation", J. Reine Angew. Math., 194 (1955), 66-87.

[12] G.S. Jones, "Asymptotic behavior and periodic solutions of a nonlinear differential-difference equation", Proc. Nat. Acad. Sci. U.S.A., 47 (1961), 879-882.

[13] Дж. Хейл, Теория функиионально-дифференииальных уравнений, Мир, М., 1984, 421 с.; пер. с англ.: J. Hale, Theory of functional differential equations, 2nd ed., Appl. Math. Sci., 3, New York-Heidelberg, Springer-Verlag, 1977, x+365 pp.

[14] А. Ю. Колесов, Н.Х. Розов, Инвариантные торы нелинейных волновых уравнений, Физматлит, М., 2004, 408 с.

[15] Е.Ф. Мищенко, В.А. Садовничий, А. Ю. Колесов, Н. Х. Розов, Автоволновые процессы в нелинейных средах с диффузией, Физматлит, М., 2005, 432 с.

\section{А. Ю. Колесов (А. Yu. Kolesov)}

Ярославский государственный университет

им. П. Г. Демидова

E-mail: kolesov@uniyar.ac.ru

\section{H. Х. Розов (N. Kh. Rozov)}

Московский государственный университет

им. М. В. Ломоносова

E-mail: rozov@rozow.mccme.ru
Поступила в редакцию 14.06 .2011 